\title{
CONCENTRATED CYCLIC ACTIONS OF HIGH PERIODICITY
}

\author{
DANIEL BEREND AND GABRIEL KATZ
}

\begin{abstract}
The class of concentrated periodic diffeomorphisms $g: M \rightarrow M$ is introduced. A diffeomorphism is called concentrated if, roughly speaking, its normal eigenvalues range in a small (with respect to the period of $g$ and the dimension of $M$ ) arc on the circle. In many ways, the cyclic action generated by such a $g$ behaves on the one hand as a circle action and on the other hand as a generic prime power order cyclic action. For example, as for circle actions, $\operatorname{Sign}(g, M)=\operatorname{Sign}\left(M^{g}\right)$, provided that the left-hand side is an integer; as for prime power order actions, $g$ cannot have a single fixed point if $M$ is closed. A variety of integrality results, relating to the usual signatures of certain characteristic submanifolds of the regular neighbourhood of $M^{g}$ in $M$ to $\operatorname{Sign}(g, M)$ via the normal $g$-representations, is established.
\end{abstract}

\section{INTRODUCTION}

This paper is an extension of a previous work [BK] dealing with a reinterpretation and refinement of the Atiyah-Singer $G$-signature theorem [AS]. We introduce and investigate a class of smooth cyclic actions behaving as circle actions with respect to a variety of properties, connecting signature-type invariants of the neighbourhood of the fixed-point set with the $G$-signature of the manifold itself. As an example of such a relation, let us mention the following nice result due to $[\mathrm{AH}]$. Given a finite rational $S^{1}$-Poincare complex $X\left(S^{1}\right.$ is the circle group), one has

$$
\operatorname{Sign}(X)=\operatorname{Sign}\left(X^{S^{1}}\right),
$$

where the $S^{1}$-fixed point set is appropriately oriented. (The special case of an $S^{1}$-rational homology manifold $X$ was discovered earlier [KR].) It will be subsequently shown (Theorems 4.31 and 4.20) that the same holds for smooth actions of an arbitrary unbounded torsion subgroup $G$ of $S^{1}$ (take $\mathbf{Q} / \mathbf{Z}$ as a model), provided that the action is linearizable at $X^{G}$. Moreover, a similar result is also valid for the class of the so-called concentrated periodic diffeomorphisms

Received by the editors December 20, 1988 .

1980 Mathematics Subject Classification (1985 Revision). Primary 57S17.

The authors' research was partially supported by the Basic Research Foundation of the Israel Academy of Sciences and Humanities grant No. 111/87, and by the United States-Israel Binational Science Foundation grant No. 86-00208. 
$g: M \rightarrow M$ with integral valued $g$-signature. Roughly speaking, a diffeomorphism $g$ of period $m$ is concentrated if the eigenvalues of the $g$-action on the normal bundle $\nu\left(M^{g}, M\right)$, are concentrated, i.e., lie in a "small" (relative to $\operatorname{dim} M$ and $m$ ) range (see Definitions 4.1-4.2). The avoid the technicalities of the precise definition, one may think of concentrated actions as those satisfying

$$
\frac{1}{2} \operatorname{dim} M \cdot \sum_{j \in J}|j|<\varphi(m) .
$$

Here $\left\{\lambda^{j}\right\}_{j \in J}, \lambda=\exp (2 \pi i / m)$, are the (normal) eigenvalues of $g_{*}: \nu\left(M^{g}, M\right)$ $\rightarrow \nu\left(M^{g}, M\right)$ and $\varphi$ is Euler's totient function.

The reformulation of the $g$-signature theorem in [BK] makes it possible to "separate" its number-theoretical and topological ingredients. These topological ingredients, called the quasi-signatures of $\nu\left(M^{g}, M\right)$, should intuitively be thought of as the usual signatures of certain characteristic submanifolds of $M^{g}$, constructed by means of $\nu\left(M^{g}, M\right)$ (see $\S 1$ for the accurate definitions). It is worthwhile mentioning that $\operatorname{Sign}\left(M^{g}\right)$ is one of these quasi-signatures.

For concentrated diffeomorphisms, the separation above enables us to replace the number-theoretical analysis in the cyclotomic field $\mathbf{Q}(\lambda)$ by similar analysis in the field of rational functions over $\mathbf{Q}$, which turns out to be much simpler. Such a replacement of the field has no effect on the normal quasi-signatures. Thus we are able to derive various results, relating the quasisignatures of $\nu\left(M^{g}, M\right)$ with the $g$-signature $\operatorname{Sign}(g, M)$ via the normal eigenvalues $\left\{\lambda^{j}\right\}_{j \in J}$ (or more accurately, via the corresponding $j$ 's) and their multiplicities. For example, we show that if a concentrated $g$-action is such that all $j \in J$ are odd and $\operatorname{Sign}(g, M) \in \mathbf{Z}$, then (see Theorem 4.21)

$$
\operatorname{Sign}(g, M)=\operatorname{Sign}\left(M^{g} \pitchfork M^{g}\right) .
$$

Here $M^{g} \pitchfork M^{g}$ denotes the bordism class of the transversal self-intersection of $M^{g}$ in $M$. In particular, if the Euler class of $\nu\left(M^{g}, M\right)$ vanishes for such an action, so does $\operatorname{Sign}(g, M)$. Theorem 4.22 and Corollaries 4.24-4.26, 4.28-4.30 present more sophisticated results of a similar nature.

It is interesting to observe a systematic similarity between a variety of modulo (prime) $p$ results obtained in $[\mathrm{BK}]$ for general diffeomorphisms of period $m=p^{e}$ and the parallel integrality results in this paper, valid for strongly concentrated diffeomorphism of arbitrary periods (see, for example, the discussion following Theorem 4.20 and also Remark 4.27). For instance, strongly concentrated diffeomorphisms (as well as general diffeomorphisms of an odd prime power period) of closed manifolds cannot have exactly one fixed point (Corollary 4.28). Similarly, a smooth action of an unbounded torsion subgroup of $S^{1}$, assumed to be linearizable at the fixed point set, cannot have a single fixed point.

Given a $g$-action of period $m$, consider the representation of $\operatorname{Sign}(g, M)$ as $\sum_{j=0}^{\mu} s_{j} \lambda^{j}$ with $\mu<\varphi(m)$ for suitable integers $\left\{s_{j}\right\}$. The $g$-signature formula in the form stated in $[\mathrm{BK}]$ suggests that there might be some correlation 
between the distribution of the $j$ 's with $s_{j} \neq 0$ in $[0, \varphi(m))$ and the distribution of the $j$ 's in $(-m / 2, m / 2]$ enumerating the normal eigenvalues $\left\{\lambda^{j}\right\}_{j \in J}$ of $g_{*}: \nu\left(M^{g}, M\right) \rightarrow \nu\left(M^{g}, M\right)$. Indeed, we prove (Corollary 4.18) that for any orientation-preserving diffeomorphism $g: M \rightarrow M$, with $\nu\left(M^{g}, M\right)$ admitting an equivariant complex structure, one has

$$
\sum_{j \in J}|j| \geq 2(\varphi(m)-\mu) / \operatorname{dim} M
$$

provided $\operatorname{Sign}(g, M) \in \mathbf{Z}[\lambda] \backslash \mathbf{Z}$ (or, equivalently, $\mu \neq 0$ ). Thus, if $\operatorname{Sign}(g, M)$ is "concentrated", i.e., $\mu$ is relatively small, yet nonzero, then $\sum_{j \in J}|j|$ ought to be relatively big. In other words, if for some diffeomorphism the inverse inequality holds, then $\operatorname{Sign}(g, M)$ is forced to take integer values.

Let us now describe briefly the plan of the paper. In $\S 1$ we present some results from $[\mathrm{BK}]$ needed in the sequel.

In $\S 2$ we investigate the class of polynomials (over the field $\mathbf{Q}$ ) in the variables $\left\{X_{j}\right\}_{j \in J}$ vanishing under the rational substitution $\left\{X_{j}=\left(\Lambda^{j}+1\right) /\left(\Lambda^{j}-1\right)\right\}_{j \in J}$, $\Lambda$ being a formal variable. These polynomials, called $J$-diagonal polynomials, possess a number of interesting properties, useful for our later applications.

$\S 3$ is devoted to a discussion of the connection between the $J$-diagonal polynomials and the algebraic numbers $\alpha_{j}=\left(\lambda^{j}+1\right) /\left(\lambda^{j}-1\right), j \in J$, which constitute the main number-theoretical ingredient of the $g$-signature formula as presented in [BK]. The principal observation here is that the "simplest" relations among the $\alpha_{j}$ 's give rise to $J$-diagonal polynomials (of course, the converse is trivial).

As shown in $[\mathrm{BK}], \operatorname{Sign}(g, M)$ is the value of a special integral polynomial $\mathscr{N}_{(M, g)} \in \mathbf{Z}\left[\left\{X_{j}\right\}_{j \in J}\right]$ at the point $\left\{\alpha_{j}\right\}_{j \in J}$. This polynomial is constructed employing only the quasi-signatures of $\nu\left(M^{g}, M\right)$ and the normal (complex) $g$-representations $\{\psi\}$. In $\S 4$ we deal with the category of concentrated cyclic actions. The basic idea is to treat $\mathcal{N}_{(M, g)}$ modulo the ideal of $J$-diagonal polynomials, taking advantage of their special properties. This enables us to investigate the question to what extent the invariant $\operatorname{Sign}(g, M)$ and the list of (complex) slice-types $\{\psi\}$ determine the normal quasi-signatures (see Proposition 4.8, Corollaries 4.9-4.10, Theorem 4.11). In particular, it is proved (Corollary 4.13) that, within our category, if $\operatorname{codim}\left(M^{g}, M\right)=2$, then this data uniquely determines the invariants

$$
\operatorname{Sign}\left(\pitchfork^{k} M_{\psi}^{g}\right)+(-1)^{d} \operatorname{Sign}\left(\pitchfork^{k} M_{\bar{\psi}}^{g}\right), \quad k=1,2, \ldots, d .
$$

Here $\pitchfork^{k} X$ denotes the $k$-fold transversal self-intersection of a submanifold $X$ in $M, M_{\psi}^{g} \subseteq M^{g}$ is the set of points of slice-type $\psi, d=(\operatorname{dim} M) / 2$, and $\bar{\psi}$ is the complex conjugate of $\psi$.

The rest of $\S 4$ consists of integrality results, some of which are already mentioned above, for actions satisfying $\operatorname{Sign}(g, M) \in \mathbf{Z}$ (this is certainly the case for homologically trivial actions and for strongly concentrated ones). 
We get these propositions by evaluating the $J$-diagonal polynomial $\mathscr{N}_{(M, g)}-$ $\operatorname{Sign}(g, M)$ at appropriately chosen points of a certain rational algebraic curve in $\mathbf{C}^{J}$. This curve, defined in terms of $J$ only, is constructed in $\S 2$. We conclude $\S 4$ with the observation that all our techniques are applicable to quite general smooth actions (see the definition preceding Theorem 4.31) of arbitrary unbounded torsion subgroups $G$ of $S^{1}$ (see Theorem 4.31). The only extra assumption is that the $G$-action is linearizable at $M^{G}$. Such an action can be approximated by a strongly concentrated cyclic action. Using this fact we show, for example, that any smooth $G$-action on $M$ necessarily has a fixed point, provided that $\operatorname{Sign}(M) \neq 0$. If this action is, in addition, linearizable at $M^{G}$, then it has at least $|\operatorname{Sign}(M)|$ fixed points; if $\operatorname{Sign}= \pm 1$, then the action has at least three fixed points. It seems that actions of dense proper subgroups of $S^{1}$, due to their ambivalent nature, linking the realms of finite and continuous groups, are quite promising objects for study.

\section{Calculating the g-Signature in terms of the NORMAL QUASI-SIGNATURES}

We shall state in this section a few results from [BK] which will be subsequently important. Let us start with notation. Let $g$ denote an arbitrary fixed generator of a cyclic group $C_{m}$ of order $m$, and $M$ a $2 d$-dimensional closed oriented smooth manifold. Suppose $g$ acts on $M$ by an orientation-preserving diffeomorphism of period $m$. The $g$-signature of $M$ is denoted by $\operatorname{Sign}(g, M)$ [AS]. Throughout the paper we assume that the normal bundle $\nu\left(M^{g}, M\right)$ admits a $g$-equivariant complex structure (if $m$ is odd this condition is automatically fulfilled). This complex structure is used to orient $\nu=\nu\left(M^{g}, M\right)$. Denote by $\{\psi\}$ the collection of complex slice-types $\psi$ of points in $M^{g}$, and by $M_{\psi}^{g}$ the set of points of slice-type $\psi$. Put $d_{\psi}=\left(\operatorname{dim} M_{\psi}^{g}\right) / 2$. Let $\nu_{\psi}=\nu\left(M_{\psi}^{g}, M\right)$ be the bundle normal to $M_{\psi}^{g}$ in $M$.

Each $\psi$ provides us with the list $\left\{\lambda^{j}\right\}_{j \in[\psi]}(\lambda=\exp (2 \pi i / m))$ of the distinct eigenvalues of the matrix $\psi(g)$ and with the list of corresponding multiplicities $\left\{n_{j}(\psi)\right\}_{j \in[\psi]}$. Normalize $[\psi]$ in such a way that $[\psi] \subseteq(-m / 2, m / 2]$. Associate with $\psi$ the set $\Omega(\psi)=\prod_{j \in[\psi]} S^{n_{j}(\psi)} \mathbf{Z}_{+}$, where $\mathbf{Z}_{+}$is the set of nonnegative integers and $S^{n} \mathbf{Z}_{+}$stands for the $n$th symmetric power of $\mathbf{Z}_{+}$. For $\omega \in \Omega(\psi)$ and $j \in[\psi]$ define the new "vector" ${ }_{j} \omega \in S^{n_{j}(\psi)} \mathbf{Z}_{+} \subseteq \Omega(\psi)$ as the "projection" of $\omega$ on $S^{n_{j}(\psi)} \mathbf{Z}_{+}$. Let $\|\omega\|$ be the $l^{1}$-norm of any vector $\tilde{\omega} \in \prod_{j \in[\psi]} \mathbf{z}_{+}^{n_{j}(\psi)}$ lying over $\omega$, and let $|\omega|$ be the number of nonzero components of $\omega$. Put

$$
\Omega\left(\psi ; d_{\psi}\right)=\left\{\omega \in \Omega(\psi):\|\omega\| \leq d_{\psi},\|\omega\| \equiv d_{\psi}(\bmod 2)\right\} .
$$

We are now able to state

Theorem A [BK, Theorem 2.2, Proposition 2.1]. Let $g: M \rightarrow M$ be an orientation-preserving diffeomorphism of period $m$. Assume that the normal 
bundle $\nu\left(M^{g}, M\right)$ admits a g-equivariant complex structure. Then

$$
\operatorname{Sign}(g, M)=\sum_{\psi} \sum_{\omega \in \Omega(\psi)}(-1)^{d_{\psi}}\left[\prod_{j \in[\psi]} \alpha_{j}^{n_{j}(\psi)+\left\|_{j} \omega\right\||-2|_{j} \omega \mid}\left(\alpha_{j}^{2}-1\right)^{\left.\right|_{j} \omega \mid}\right] \mathscr{S}_{\omega}\left(\nu_{\psi}\right) \text {. }
$$

Here the rational integers $\left\{\mathscr{S}_{\omega}\left(\nu_{\psi}\right)\right\}_{\omega \in \Omega(\psi)}$, called the quasi-signatures of the normal bundle $\nu_{\psi}=\nu\left(M_{\psi}^{g}, M\right)$, are defined in terms of the characteristic class $T_{\omega}\left(\nu_{\psi}\right) \in H^{2 *}\left(M_{\psi}^{g} ; \mathbf{Q}\right)$ of $\nu_{\psi}[\mathrm{BK},(2.5)]$ by the formula

$$
\mathscr{S}_{\omega}\left(\nu_{\psi}\right)=\left\langle T_{\omega}\left(\nu_{\psi}\right) \cdot L\left(M_{\psi}^{g}\right),\left[M_{\psi}^{g}\right]\right\rangle,
$$

$L\left(M_{\psi}^{g}\right)$ being the Hirzebruch L-class of the tangent bundle of $M_{\psi}^{g}$ as defined in $[\mathrm{H}]$.

The integers $\left\{\mathscr{S}_{\omega}\left(\nu_{\psi}\right)\right\}_{\omega, \psi}$ are invariants of the class of $M$ in the group of equivariant oriented bordisms.

In (1.1) the orientation $M_{\psi}^{g}$ is induced by the orientations of $M$ and $\nu_{\psi}$. Note that a straightforward dimension argument shows that $\mathscr{S}_{\omega}\left(\nu_{\psi}\right)=0$ when either $\|\omega\|>d_{\psi}$ or $\|\omega\| \not \equiv d_{\psi}(\bmod 2)$. Consequently, the summation over $\omega \in \Omega(\psi)$ in the $g$-signature formula of Theorem A can be replaced by summation over $\omega \in \Omega\left(\psi ; d_{\psi}\right)$.

If $\nu_{\psi}$ splits equivariantly as $\bigoplus_{j \in[\psi]}\left(\bigoplus_{l=1}^{n_{j}(\psi)}\left(\nu_{\psi}\right)_{j, l}\right)$ into a direct sum of complex 1-dimensional bundles $\left(\nu_{\psi}\right)_{j, l}$, then the $\mathscr{S}_{\omega}\left(\nu_{\psi}\right)$ 's may be interpreted in a quite more geometric fashion as follows:

$$
\mathscr{S}_{\omega}\left(\nu_{\psi}\right)=\left|\mathrm{St}_{\tilde{\omega}}\right|^{-1} \sum_{\sigma \in S(\mathbf{n}(\psi))} \operatorname{Sign}\left(M_{\psi}^{g} \pitchfork_{\sigma \tilde{\omega}} M_{\psi}^{g}\right),
$$

where $S(k)$ is the symmetric group on $k$ symbols, $S(\mathbf{n})=\prod_{j} S\left(n_{j}\right), \mathrm{St}_{\tilde{\omega}}$ is the stabilizer of $\tilde{\omega}=\left\{\tilde{\omega}_{j, l}\right\}$ under the natural $S(\mathbf{n}(\psi))$-action, and $M_{\psi}^{g} \pitchfork_{\sigma \tilde{\omega}} M_{\psi}^{g}$ denotes the bordism class of the transversal self-intersection of $M_{\psi}^{g}$ in the space of an auxiliary bundle $\bigoplus_{j, l}(\sigma \tilde{\omega})_{j, l}\left(\nu_{\psi}\right)_{j, l}$ constructed by means of $\omega$. In fact, comparing (1.1) with (1.2), one realizes that $\mathscr{S}_{\omega}\left(\nu_{\psi}\right)$ depends neither on the equivariant splitting of $\nu_{\psi}$ nor on the choice of $\tilde{\omega}$.

Note that the $\omega$ 's having identical $\left\|_{j} \omega\right\|$ 's and $\left.\right|_{j} \omega \mid$ 's, $j \in[\psi]$, give rise to the same monomials $\prod_{j \in[\psi]} \alpha_{j}^{n_{j}(\psi)+\left\|_{j} \omega\right\|-\left.2\right|_{j} \omega \mid}\left(\alpha_{j}^{2}-1\right)^{\left|l_{j} \omega\right|}$ in the $\alpha_{j}$ 's and $\left(\alpha_{j}^{2}-1\right)$ 's in the $g$-signature formula of Theorem A.

Set $b_{j}=n_{j}(\psi)+\left\|_{j} \omega\right\|-\left.2\right|_{j} \omega \mid$ and $c_{j}=\left.\right|_{j} \omega \mid$. It is readily verified that the set $\mathscr{O}_{\psi}=\left\{(\mathbf{b}, \mathbf{c})=\left(\left\{b_{j}\right\},\left\{c_{j}\right\}\right)_{j \in[\psi]}: \omega \in \boldsymbol{\Omega}\left(\psi ; d_{\psi}\right)\right\}$ is the finite subset of $\mathbf{Z}_{+}^{[\psi]} \times \mathbf{Z}_{+}^{[\psi]}$ determined by the constraints:

$$
\begin{cases}c_{j} \leq n_{j}(\psi) \leq b_{j}+c_{j}, & j \in[\psi], \\ c_{j}=0 \Rightarrow b_{j}=n_{j}(\psi), & j \in[\psi], \\ \|\mathbf{b}+2 \mathbf{c}\| \leq d, & \|\mathbf{b}\| \equiv d(\bmod 2)(d=(\operatorname{dim} M / 2)) .\end{cases}
$$


With these notations the $g$-signature formula takes the form

$$
\operatorname{Sign}(g, M)=\sum_{\psi} \sum_{(\mathbf{b}, \mathbf{c}) \in \mathscr{O}_{\psi}}\left[\prod_{j \in[\psi]} \alpha_{j}^{b_{j}}\left(\alpha_{j}^{2}-1\right)^{c_{j}}\right] a_{\mathbf{b}, \mathbf{c}}\left(\nu_{\psi}\right),
$$

where the characteristic number $a_{\mathbf{b}, \mathbf{c}}\left(\nu_{\psi}\right)=(-1)^{d_{\psi}} \sum_{\omega} \mathscr{S}_{\omega}\left(\nu_{\psi}\right)$, the summation extending over the set

$$
\left\{\omega \in \Omega\left(\psi, d_{\psi}\right):\left\|_{j} \omega\right\|=b_{j}+2 c_{j}-n_{j}(\psi),\left.\right|_{j} \omega \mid=c_{j} \forall j \in[\psi]\right\} .
$$

Let $J=\bigcup_{\psi}[\psi]$. Consider the polynomial in $\mathbf{Z}\left[\left\{X_{j}\right\}_{j \in J}\right]$ defined by

$$
\begin{aligned}
\mathscr{N}_{(M, g)}\left(\left\{X_{j}\right\}\right)=\sum_{\psi} \sum_{\omega \in \Omega(\psi)}( & (-1)^{d_{\psi}} \mathscr{S}_{\omega}\left(\nu_{\psi}\right) \\
\times & {\left[\prod_{j \in[\psi]} X_{j}^{n_{j}(\psi)+||\left\|_{j} \omega\right\|-\left.2\right|_{j} \omega \mid}\left(X_{j}^{2}-1\right)^{\left.\right|_{j} \omega \mid}\right], }
\end{aligned}
$$

or, equivalently, by

$$
\mathscr{N}_{(M, g)}\left(\left\{X_{j}\right\}_{j \in J}\right)=\sum_{\psi} \sum_{(\mathbf{b}, \mathbf{c}) \in \mathscr{O}_{\psi}} a_{\mathbf{b}, \mathbf{c}}\left(\nu_{\psi}\right)\left[\prod_{j \in[\psi]} X_{j}^{b_{j}}\left(X_{j}^{2}-1\right)^{c_{j}}\right] .
$$

By Theorem A, this polynomial, evaluated at the point $\left\{\alpha_{j}\right\}_{j \in J}$, gives $\operatorname{Sign}(g, M)$. Since $\alpha_{-j}=-\alpha_{j}$, we may view $\mathcal{N}_{(M, g)}$ as an element of $\mathrm{Z}\left[\left\{X_{j}\right\}_{j \in J}\right] / I$, where $I$ is the ideal generated by $\left\{X_{j}+X_{-j}: j,-j \in J\right\}$.

Prior to studying the polynomial $\mathscr{N}_{(M, g)}$ (for general actions) we turn to investigate a class of so-called $J$-diagonal polynomials which will be useful for understanding $\mathcal{N}_{(M, g)}$.

\section{2. $J$-DiAgONAL POLYNOMIALS}

Let $K$ be a field of characteristic 0 . We consider $\mathbf{Q}$ as a subfield of $K$. Let $J$ stand for a (finite) subset of $\mathbf{Z} \backslash\{0\}$.

Definition 2.1. A polynomial $P \in K\left[\left\{X_{j}\right\}_{j \in J}\right]$ is $J$-diagonal if it vanishes under the rational substitution $X_{j}=\left(\Lambda^{j}+1\right) /\left(\Lambda^{j}-1\right), j \in J, \Lambda$ being a formal variable.

The set of all $J$-diagonal polynomials obviously forms an ideal of $K\left[\left\{X_{j}\right\}_{j \in J}\right]$ denoted by $D_{K}(J)$.

Example 2.2. Let $j_{1}, j_{2}, \ldots, j_{r}$ be a finite sequence in $J$ with $\sum_{i=1}^{r} j_{i}=0$. The integral polynomial

$$
P_{j_{1}, j_{2}, \ldots, j_{r}}=\left[\prod_{i=1}^{r}\left(X_{j_{i}}+1\right)-\prod_{i=1}^{r}\left(X_{j_{i}}-1\right)\right] / 2
$$

of degree $r-1$ is readily verified to be $J$-diagonal. In particular, if $j,-j \in J$, then the polynomial $P_{j,-j}=X_{j}+X_{-j}$ is $J$-diagonal. Another special case 
is that of the quadratic polynomial $P_{j_{1}, j_{2}, j_{3}}=X_{j_{1}} X_{j_{2}}+X_{j_{1}} X_{j_{3}}+X_{j_{2}} X_{j_{3}}+1$, provided that $j_{1}, j_{2}, j_{3} \in J$ and $j_{3}=-\left(j_{1}+j_{2}\right)$.

Denote by $\Delta(j)$ the sign of $j \in \mathbf{Z}$. For given $j, k \in J$ put $j^{\prime}=|j /(j, k)|$ and $k^{\prime}=|k /(j, k)|$. The polynomial

$$
R_{j, k}=\left[\left(X_{j}+\Delta(j)\right)^{k^{\prime}} \cdot\left(X_{k}-\Delta(k)\right)^{j^{\prime}}-\left(X_{j}-\Delta(j)\right)^{k^{\prime}} \cdot\left(X_{k}+\Delta(k)\right)^{j^{\prime}}\right] / 2
$$

is another important example of a $J$-diagonal polynomial. (In fact, for $\Delta(j)=$ $\Delta(k), R_{j, k}=P_{j, \ldots, j, k, \ldots, k}$ with suitable multiplicities of the $j$ 's and $k$ 's, and for $\Delta(j)=\Delta(k)$ one can obtain $R_{j, k}$ by symmetrizing $J$ with respect to the involution $j \rightarrow-j$ and employing $P_{j,-j}$.)

Question. For symmetric $J$, does the ideal generated by all polynomials $P_{j_{1}, \ldots, j_{r}}$ coincide with $D_{K}(J)$ ? Is it true that any quadratic $J$-diagonal polynomial is a linear combination of $P_{j_{1}, j_{2}, j_{3}}$ 's?

Let $\mathscr{L}_{K}(J) \subseteq K^{J}$ be the affine variety defined by the equations $\left\{R_{j, k}=0\right\}_{j, k \in J}$.

Lemma 2.3. $\mathscr{L}_{K}(J)$ is a curve in $K^{J}$, birationally isomorphic to the line $K^{1}$, and smooth away from the two points $A=\left\{\Delta(j)_{j \in J}\right\}$ and $B=-A$. The ideal $D_{K}(J)$ of $J$-diagonal polynomials is the zero ideal $I_{\mathscr{L}_{K}(J)}$ of the curve $\mathscr{L}_{K}(J)$.

Proof. We shall assume that the greatest common divisor of all elements of $J$ is 1 (this is really the case we need for geometric applications: moreover, the argument for general $J$ 's is a minor modification of the subsequent one). Define a rational map $\theta: K^{1} \rightarrow K^{J}$ by $\theta(q)=\left\{\left(q^{j}+1\right) /\left(q^{j}-1\right)\right\}_{j \in J}, q \in K$. This mapping is properly defined on $\left\{q \in K: q^{j} \neq 1 \forall j \in J\right\}$. It is easy to check that $\theta\left(K^{1}\right) \subseteq \mathscr{L}_{K}(J)$. Write $1=\sum_{j \in J} j \cdot u_{j}$ for appropriately chosen integers $u_{j}$, and define $\theta^{-1}: K^{J} \rightarrow K^{1}$ by

$$
\theta^{-1}\left(\left\{X_{j}\right\}_{j \in J}\right)=\prod_{j \in J}\left(\frac{X_{j}+1}{X_{j}-1}\right)^{u_{j}} .
$$

Note that $\theta^{-1}$ is properly defined at least in the complement of the union of the hyperplanes $\left\{X_{j}= \pm 1\right\}_{j \in J}$. The curve $\mathscr{L}_{K(J)}$ meets this union exactly at the two points $A$ and $B$. It is routinely verified that $\theta^{-1} \circ \theta=\mathrm{id}_{K^{1}}$, and the verification that $\left.\theta \circ \theta^{-1}\right|_{\mathscr{L}_{K}(J)}=\mathrm{id}_{\mathscr{L}_{K}(J)}$ is based on the fact that the rational functions $\left[\left(X_{j}+1\right) /\left(X_{j}-1\right)\right]^{k /(j, k)}$ and $\left[\left(X_{k}+1\right) /\left(X_{k}-1\right)\right]^{j /(j, k)}$ coincide on $\mathscr{L}_{K}(J)$. Thus, $\mathscr{L}_{K}(J)$ is a rational curve. An elementary analysis of the differentials of the $R_{j, k}$ 's shows that they constitute a system of maximal rank $\#(J)-1$ at each point of $\mathscr{L}_{K}(J)$ except for $A$ and $B$.

Now take $P \in D_{K}(J)$. For any $q \in K$ such that $q^{j} \neq 1$ for each $j \in$ $J$ we have $P(\theta(q))=0$. Since $K$ is infinite, there are infinitely many $q$ 's, 
and therefore infinitely many corresponding $\theta(q)$ 's. For each of these, $\theta(q) \in$ $\mathscr{L}_{K}(J)$. Thus, $P$ vanishes identically on the curve $\mathscr{L}_{K}(J)$, which means that $P \in I_{\mathscr{L}_{K}(J)}$. The converse direction is equally easy. In fact, let $P \in I_{\mathscr{L}_{K}(J)}$. Since $\theta(q) \in \mathscr{L}_{K}(J)$ for infinitely many $q$ 's, $\left.P\left(\left\{\Lambda^{j}+1\right) /\left(\Lambda^{j}-1\right)\right\}_{j \in J}\right)=0$ in $K(\Lambda)$, and hence $P \in D_{K}(J)$.

Corollary 2.4. Any J-diagonal polynomial $P \in K\left[\left\{X_{j}\right\}_{j \in J}\right]$ vanishes under the following substitutions:

(1) $X_{j}=\Delta(j), j \in J$

(2) $X_{j}=-\Delta(j), j \in J$.

Proof. In view of the preceding lemma, $P$ has to vanish at the points $A$ and $B$ of $\mathscr{L}_{K}(J)$.

Lemma 2.5. Let $l$ be a positive integer not dividing any $j \in J$, and $r$ an integer relatively prime to $l$. Then every $J$-diagonal polynomial vanishes under the substitution $X_{j}=\alpha_{j r / l}=\left(e^{2 \pi i j r / l}+1\right) /\left(e^{2 \pi i j r / l}-1\right), j \in J \quad(j r / l \in \mathbf{Q} / \mathbf{Z})$. In particular, for $l=2,3,4$ and $r= \pm 1$ the corresponding substitutions are:

(1) $l=2$ : (assuming $2 \nmid j, j \in J) X_{j}=0, j \in J$.

(2) $l=3:$ (assuming $3 \nmid j, j \in J) X_{j}= \begin{cases}\mp i \sqrt{ } 3 / 3, & j \equiv 1(\bmod 3), \\ \pm i \sqrt{ } 3 / 3, & j \equiv 2(\bmod 3) .\end{cases}$

(3) $l=4:$ (assuming $4 \nmid j, j \in J) X_{j}= \begin{cases}\mp i, & j \equiv 1(\bmod 4), \\ 0, & j \equiv 2(\bmod 4), \\ \pm i, & j \equiv 3(\bmod 4)\end{cases}$

In fact, given a $P \in D_{K}(J)$ we substitute $\Lambda=e^{2 \pi i r / l}$ in

$$
P\left(\left\{\left(\Lambda^{j}+1\right) /\left(\Lambda^{j}-1\right)\right\}_{j \in J}\right)
$$

which is legitimate under the assumptions of the lemma.

Let $P \in K\left[\left\{X_{j}\right\}_{j \in J}\right]$. Write $P\left(\left\{\left(\Lambda^{j}+1\right) /\left(\Lambda^{j}-1\right)\right\}_{j \in J}\right)=F(\Lambda) / G(\Lambda)$ with $F, G \in K(\Lambda)$ relatively prime. Put $|J|=\{|j|: j \in J\}$. Denote $\delta_{J}(P)=\operatorname{deg} F$. Letting $\operatorname{deg}_{ \pm j}(P)$ be the degree of $P$ viewed as a polynomial in $X_{j}$ and $X_{-j}$ only, it is easy to check that

$$
\delta_{J}(P) \leq \sum_{j \in|J|} j \operatorname{deg}_{ \pm j}(P) \leq\left(\sum_{j \in|J|} j\right) \operatorname{deg} P .
$$

Actually, for a $P$ with a prescribed list $\mathscr{E}$ of monomials in the $X_{j}$ 's one can find a sharper bound for $\delta_{J}(P)$. Assume that $P=\sum_{\mathbf{b} \in \mathscr{E}} a_{\mathbf{b}}\left(\prod_{j \in J} X_{j}^{b_{j}}\right)$, where $\mathbf{b}=$ $\left\{b_{j}\right\}_{j \in J}$ and $a_{\mathbf{b}} \in K$. Our immediate goal is to compute the multiplicity of the pole $\lambda_{1 / k}=e^{2 \pi i / k}, k \in \mathbf{N}$, of the rational function $\prod_{j \in J}\left[\left(\Lambda^{j}+1\right) /\left(\Lambda^{j}-1\right)\right]^{b_{j}}$. 
Using the identities

$$
\begin{aligned}
& \Lambda^{s}-1=(\Lambda-1)\left(\Lambda-\lambda_{1 / s}\right) \cdots\left(\Lambda-\lambda_{1 / s}^{s-1}\right), \\
& \Lambda^{s}+1=\left(\Lambda-\lambda_{1 / 2 s}\right)\left(\Lambda-\lambda_{1 / 2 s}^{3}\right) \cdots\left(\Lambda-\lambda_{1 / 2 s}^{2 s-1}\right),
\end{aligned}
$$

we observe that this multiplicity is, for a generic $P, \max \left(0, \chi_{k}(\mathbf{b})\right)$, where

$$
\chi_{k}(\mathbf{b})= \begin{cases}\sum_{\{l: l k \in J\}} b_{l k}, & k \equiv 1(\bmod 2), \\ \sum_{\{l: l k / 2 \in J\}}(-1)^{l} b_{l k / 2}, & k \equiv 0(\bmod 2) .\end{cases}
$$

Thus, the denominator $G(\Lambda)$ of the rational function

$$
\sum_{\mathbf{b} \in \mathscr{E}} a_{\mathbf{b}} \prod_{j \in J}\left[\left(\Lambda^{j}+1\right) /\left(\Lambda^{j}-1\right)\right]^{b_{j}}
$$

is given by

$$
\begin{aligned}
G(\Lambda) & =\prod_{k=1}^{\max |J|} \prod_{\{j:(j, k)=1\}}\left(\Lambda-\lambda_{j / k}\right)^{\max _{\mathbf{b} \in \mathscr{E}} \max \left(0, \chi_{k}(\mathbf{b})\right)} \\
& =\prod_{k=1}^{\max |J|} \Phi_{k}(\Lambda)^{\max _{\mathbf{b} \in \mathscr{E}} \max \left(0, \chi_{k}(\mathbf{b})\right)}
\end{aligned}
$$

$\Phi_{k}$ being the $k$ th cyclotomic polynomial. Consequently,

$$
\operatorname{deg} G=\sum_{k=1}^{\max |J|} \varphi(k) \cdot \max _{\mathbf{b} \in \mathscr{E}} \max \left(0, \chi_{k}(\mathbf{b})\right),
$$

where $\varphi$ is Euler's totient function. On the other hand, denoting by $\|\cdot\|$ the nonarchimedean norm on $K(\Lambda)$ defined by

$$
\|f / g\|=c^{-(\operatorname{deg} f-\operatorname{deg} g)}, \quad f, g \in K(\Lambda) \quad(0<c<1),
$$

one observes that since $\left\|\left(\Lambda^{j}+1\right) /\left(\Lambda^{j}-1\right)\right\|=1$ we have

$$
\left\|P\left(\left\{\left(\Lambda^{j}+1\right) /\left(\Lambda^{j}-1\right)\right\}_{j \in J}\right)\right\| \leq 1 .
$$

Therefore,

$$
\operatorname{deg} F \leq \operatorname{deg} G
$$

which implies that

$$
\delta_{J}(P) \leq \sum_{k=1}^{\max |J|} \varphi(k) \cdot \max _{\mathbf{b} \in \mathscr{E}} \max \left(0, \chi_{k}(\mathbf{b})\right) .
$$


This yields

Lemma 2.6. Let $P \in K\left[\left\{X_{j}\right\}_{j \in J}\right]$. If the rational function $Q(\Lambda)=$ $P\left(\left\{\left(\Lambda^{j}+1\right) /\left(\Lambda^{j}-1\right)\right\}_{j \in J}\right)$ vanishes at more than

$$
\sum_{k=1}^{\max |J|} \varphi(k) \cdot \max _{\mathbf{b} \in \mathscr{E}} \max \left(0, \chi_{k}(\mathbf{b})\right)
$$

points, then $P \in D_{K}(J)$.

Remark 2.7. It is evident from the discussion preceding Lemma 2.6 that, for generic $P$ (with prescribed $\mathscr{E}$ ), $(2.3)$ is an equality. Hence, the right-hand side of (2.3) gives the number of zeros of such a $P$ on the curve $\mathscr{L}_{C}(J)$. In particular, a generic affine hyperplane in $C^{J}$ meets $\mathscr{L}_{C}(J)$ at $\sum_{\{k: \exists j \in J, k \mid j\}} \varphi(k)$ points, which means that this number is the degree of $\mathscr{L}_{C}(J)$.

Lemma 2.8. Suppose that a polynomial $P \in K\left[\left\{X_{j}\right\}_{j \in J}\right]$ gives a polynomial $Q \in K[\Lambda]$ under the substitution $X_{j}=\left(\Lambda^{j}+1\right) /\left(\Lambda^{j}-1\right), j \in J$. Then $Q$ is a constant polynomial.

If fact, this follows readily from (2.2).

It will be useful for geometric applications to establish an analogue of (2.2) for polynomials $P$ given in the form $P=\sum_{(\mathbf{b}, \mathbf{c}) \in \mathcal{O}} a_{\mathbf{b}, \mathbf{c}}\left[\prod_{j \in J} X_{j}^{b_{j}}\left(X_{j}^{2}-1\right)^{c_{j}}\right]$. With $\chi_{\kappa}(\mathbf{b})$ as in $(2.1)$, set

$$
\tilde{\chi}_{k}(\mathbf{b}, \mathbf{c})=\chi_{k}(\mathbf{b})+2 \sum_{\{l: l k \in J\}} c_{l k} .
$$

As before we get (for generic $P$ of the above from)

$$
\operatorname{deg} G=\sum_{k=1}^{\max |J|} \varphi(k) \cdot \max _{(\mathbf{b}, \mathbf{c}) \in \mathcal{O}} \max \left(0, \tilde{\chi}_{k}(\mathbf{b}, \mathbf{c})\right)
$$

and it is easy to see that $\operatorname{deg} F \leq \operatorname{deg} G-\min \sum_{j \in J} c_{j}$. This implies

Lemma 2.9. Let $P=\sum_{(\mathbf{b}, \mathbf{c}) \in \mathcal{O}} a_{\mathbf{b}, \mathbf{c}}\left[\prod_{j \in J} X_{j}^{b_{j}}\left(X_{j}^{2}-1\right)^{c_{j}}\right]$. If the rational function $Q(\Lambda)=P\left(\left\{\left(\Lambda^{j}+1\right) /\left(\Lambda^{j}-1\right)\right\}_{j \in J}\right)$ vanishes at more than

$$
\sum_{k=1} \varphi(k) \max _{(\mathbf{b}, \mathbf{c}) \in \mathscr{Q}} \max \left(0, \tilde{\chi}_{k}(\mathbf{b}, \mathbf{c})\right)-\min _{\mathbf{c}} \sum_{j \in J} j c_{j}
$$

points, then $P \in D_{K}(J)$.

Let us present a few more properties of $J$-diagonal polynomials.

For an arbitrary $\mathbf{t} \in \mathbf{Z}^{J}$ we define the $\mathbf{t}$-weighted degree $\operatorname{deg}_{\mathbf{t}}$ for elements of $K\left[\left\{X_{j}\right\}_{j \in J}\right]$ by putting $\operatorname{deg}_{\mathrm{t}}\left(X_{j}\right)=t_{j}$ and extending it in a standard way. Denote by ${ }_{\mathrm{t}} P$ the sum of the leading $\operatorname{deg}_{\mathrm{t}}$-homogeneous terms of $P$.

We shall be interested in weights $\mathbf{t}_{l}, l \in N$, of the following form:

$$
\mathbf{t}_{l, j}= \begin{cases}(-1)^{2 j / l}, & l \mid 2 j, \\ 0, & l+2 j .\end{cases}
$$


Lemma 2.10. Let $P \in D_{K}(J)$ and $l \in \mathbf{N}$. Then the leading $\operatorname{deg}_{\mathbf{t}_{l}}$-homogeneous part ${ }_{\mathbf{t}_{l}} P$ of $P$ vanishes at the point $\gamma_{l}$ given by

In particular:

$$
\gamma_{l, j}= \begin{cases}j^{(-1)^{1-2 j / l},}, & l \mid 2 j, \\ \alpha_{j / l}, & l+2 j .\end{cases}
$$

(i) ${ }_{\mathbf{t}_{1}} P(\ldots,-1 / 3,-1 / 2,-1 ; 1,1 / 2,1 / 3, \ldots)=0$.

(ii) ${ }_{\mathbf{t}_{2}} P(\ldots,-1 / 4,-3,-1 / 2,-1 ; 1,1 / 2,3,1 / 4, \ldots)=0$.

(iii) ${ }_{\mathbf{t}_{3}} P(\ldots,-1 / 3,-i \sqrt{ } 3 / 3, i \sqrt{ } 3 / 3 ;-i \sqrt{ } 3 / 3, i \sqrt{3} / 3,1 / 3, \ldots)=0$.

(iv) ${ }_{\mathbf{t}_{4}} P(\ldots,-1 / 4,-i,-2, i ;-i, 2, i, 1 / 4, \ldots)=0$.

Proof. We have

$$
\begin{aligned}
0= & \left(\Lambda-\lambda_{1 / l}\right)^{\operatorname{deg}_{\mathrm{t}^{\prime} /}(P)} \cdot P\left(\left\{\left(\Lambda^{j}+1\right) /\left(\Lambda^{j}-1\right)\right\}\right) \\
= & \left(\Lambda-\lambda_{1 / l}\right)^{\operatorname{deg}_{t_{l}}(P)} \cdot{ }_{\mathbf{t}_{l}} P\left(\left\{\left(\Lambda^{j}+1\right) /\left(\Lambda^{j}-1\right)\right\}\right) \\
& +\left(\Lambda-\lambda_{1 / l}\right)^{\operatorname{deg}_{t_{l}}(P)} \cdot\left(P-{ }_{\mathbf{t}_{l}} P\right)\left(\left\{\left(\Lambda^{j}+1\right) /\left(\Lambda^{j}-1\right)\right\}\right) .
\end{aligned}
$$

Since $\operatorname{deg}_{\mathbf{t}_{l}}\left(P-{ }_{\mathbf{t}_{l}} P\right)<\operatorname{deg}_{\mathbf{t}_{l}}(P)$, the power to which the factor $\Lambda-\lambda_{1 / l}$ appears in the numerator of the second term on the right-hand side exceeds that to which it appears in the numerator. It follows that the latter term vanishes, under the substitution $\Lambda=\lambda_{1 / l}$, and therefore so does the former. Now this former term is the value of ${ }_{\mathbf{t}_{l}} P$ at

$$
X_{j}= \begin{cases}{\left[\left(\Lambda^{j}+1\right) /\left(\Lambda^{j}-1\right)\right]\left(\Lambda-\lambda_{1 / l}\right)^{(-1)^{2 j / l},},} & l \mid 2 j, \\ \left(\Lambda^{j}+1\right) /\left(\Lambda^{j}-1\right), & l \nmid 2 j .\end{cases}
$$

Since

$$
\lim _{\lambda \rightarrow \lambda_{1 / l}} X_{j}= \begin{cases}\left(j / 2 \lambda_{1 / l}\right)^{(-1)^{1+2 j / l},}, & l \mid 2 j, \\ \alpha_{j / l}, & l+2 j,\end{cases}
$$

the $\operatorname{deg}_{\mathbf{t}_{l}}$-homogeneity of $\mathbf{t}_{l} P$ shows that it vanishes at $\gamma_{l}$.

Corollary 2.11. Any J-diagonal polynomial of the form $\sum_{j \in J} P_{j}\left(X_{j}\right)$, where $P_{j} \in K\left[X_{j}\right]$ for $j \in J$, becomes identically 0 under the substitution $X_{j} \rightarrow$ $-X_{-j}, j<0, j,-j \in J$. In particular, any linear $J$-diagonal polynomial is a linear combination of the $\left(X_{j}+X_{-j}\right)$ 's with $j,-j \in J$.

In fact, suppose that $P \in D_{K}(J)$. Let $l=\max \left\{|j|: j \in J, P_{j}\left(X_{j}\right)+P_{-j}\left(-X_{j}\right)\right.$ is nonconstant $\}$. Applying Lemma 2.10 with this $l$, we get a contradiction.

3. RELATIONS AMONG THE $\alpha_{j}$ 's AND THEIR CONNECTION WITH $J$-DIAGONAL POLYNOMIALS

For a given $J$ and $m>\max |J|$, consider the ring homomorphism

$$
\nabla_{J}: K\left[\left\{X_{j}\right\}_{j \in J}\right] \rightarrow K\left[\left\{\alpha_{j / m}\right\}_{j \in J}\right]
$$


carrying 1 to 1 and $X_{j}$ to $\alpha_{j / m}, j \in J$. As mentioned earlier, $\nabla_{J}$ annihilates the ideal generated by $\left\{X_{j}+X_{m-j}: j, m-j \in J\right\}$.

A description of $\operatorname{Ker} \nabla_{J}$ (in terms of some minimal set of generators) would be important for understanding the number theory related to the $g$-signature formula of Theorem A. Unfortunately, such a goal does not seem to be easily accessible.

Nevertheless, for elements $P \in K\left[\left\{X_{j}\right\}_{j \in J}\right]$ which are in some sense of a small degree relative to $\varphi(m), P$ lies in $\operatorname{Ker} \nabla_{J}$ iff $P$ is $J$-diagonal. Note that in any case $\operatorname{Ker} \nabla_{J} \supseteq D_{K}(J)$. We shall presently make these observations more accurate.

Let $\sigma$ be an element of the Galois group $(\mathbf{Z} / m \mathbf{Z})^{*}$ of $\mathbf{Q}\left(\lambda_{1 / m}\right) / \mathbf{Q} . \quad \sigma$ induces a ring homomorphism $\sigma^{*}: K\left[\left\{X_{j}\right\}_{j \in J}\right] \rightarrow K\left[\left\{X_{\sigma j}\right\}_{j \in J}\right]$ given by $\sigma^{*}(1)=$ 1 and $\sigma^{*}\left(X_{j}\right)=X_{\sigma j}, j \in J$. Obviously, $P \in \operatorname{Ker} \nabla_{J}$ implies $\sigma^{*} P \in \operatorname{Ker} \nabla_{\sigma J}$. Combining this with Lemma 2.3, one gets

Lemma 3.1. Let $P \in K\left[\left\{X_{j}\right\}_{j \in J}\right]$, and assume that $\delta_{\sigma J}\left(\sigma^{*} P\right)<\varphi(m)$ for a certain $\sigma \in(\mathbf{Z} / m \mathbf{Z})^{*}$. Then the following are equivalent:

(i) $P \in \operatorname{Ker} \nabla_{J}$.

(ii) $\sigma^{*} P$ is $\sigma J$-diagonal.

(iii) $\sigma^{*} P$ belongs to the zero ideal $I_{\mathscr{L}_{K}(\sigma J)}$ of the rational curve $\mathscr{L}_{K}(\sigma J)$ (defined by the equations $\left\{R_{j, k}=0\right\}_{j, k \in \sigma J}$ ).

(iv) There exist $T+1$ distinct positive rational numbers $\left\{q_{r}\right\}_{0 \leq r \leq T}$ such that $P\left(\left\{\left(q_{r}^{\sigma j}+1\right) /\left(q_{r}^{\sigma j}-1\right)\right\}_{j \in J}\right)=0,0 \leq r \leq T$, where $T \geq \delta_{\sigma J}\left(\sigma^{*} P\right)$.

Employing Lemmas 2.6 and 2.9, this yields

Corollary 3.2. (a) Let $P=\sum_{\mathbf{b} \in \mathscr{E}} \mathbf{a}_{\mathbf{b}} \prod_{j \in J} X_{j}^{b_{j}}$. If

$$
\sum_{k=1}^{\max |\sigma J|} \varphi(k) \cdot \max _{\mathbf{b} \in \mathscr{E}} \max \left(0, \chi_{k}\left(\sigma^{-1} \mathbf{b}\right)\right)<\varphi(m)
$$

for some $\sigma \in(\mathbf{Z} / m \mathbf{Z})^{*}$, then properties (i)-(iv) in Lemma 3.1 are equivalent, with $T$ being taken as the left-hand side of (3.1).

(b) Let $P=\sum_{(\mathbf{b}, \mathbf{c}) \in \mathscr{O}} \mathbf{a}_{\mathbf{b}, \mathbf{c}} \prod_{j \in J} X_{j}^{b_{j}}\left(X_{j}^{2}-1\right)^{c_{j}}$. If

$$
\prod_{k=1}^{\max |\sigma J|} \varphi(k) \cdot \max _{(\mathbf{b}, \mathbf{c}) \in \mathscr{O}} \max \left(0, \tilde{\chi}_{k}\left(\sigma^{-1} \mathbf{b}, \sigma^{-1} \mathbf{c}\right)\right)-\min _{\mathbf{c}} \sum_{j \in \sigma J}|j| \mathbf{c}_{\sigma^{-1} j}<\varphi(m)
$$

for some $\sigma \in(\mathbf{Z} / m \mathbf{Z})^{*}$, then properties (i)-(iv) in Lemma 3.1 are equivalent, with $T$ being taken as the left-hand side of (3.2).

Lemma 3.3. Let $P=\sum_{\mathbf{b} \in \mathscr{\mathscr { C }}} a_{\mathbf{b}} \prod_{j \in J} X_{j}^{b_{j}} \in \mathbf{Q}\left[\left\{X_{j}\right\}_{j \in J}\right]$.

Write $P\left(\left\{\alpha_{j / m}\right\}_{j \in J}\right)=Q\left(\lambda_{1 / m}\right)$ with $Q \in \mathbf{Q}[\Lambda], \operatorname{deg} Q<\varphi(m)$. If

$$
\sum_{k=1}^{\max |J|} \varphi(k) \cdot \max _{\mathbf{b} \in \mathscr{E}} \max \left(0, \chi_{k}(\mathbf{b})\right)<\varphi(m)-\operatorname{deg} Q,
$$

then $Q$ is constant and $P-Q \in D_{\mathbf{Q}}(J)$. 
An analogous statement may be formulated for polynomials of the form

$$
P=\sum_{(\mathbf{b}, \mathbf{c}) \in \mathscr{O}} a_{\mathbf{b}, \mathbf{c}} \prod_{j \in J} X_{j}^{b_{j}}\left(X_{j}^{2}-1\right)^{c_{j}} .
$$

Proof. Put $P\left(\left\{\left(\Lambda^{j}+1\right) /\left(\Lambda^{j}-1\right)\right\}_{j \in J}\right)=F(\Lambda) / G(\Lambda)$, with $F, G \in \mathbf{Q}[\Lambda]$ relatively prime. The equality $P\left(\left\{\alpha_{j / m}\right\}_{j \in J}\right)=Q\left(\lambda_{1 / m}\right)$ yields $F\left(\lambda_{1 / m}\right)-$ $G\left(\lambda_{1 / m}\right) \cdot Q\left(\lambda_{1 / m}\right)=0$. By $(2.2)$ we get

$$
\operatorname{deg}(F-G \cdot Q) \leq \max (\operatorname{deg} F, \operatorname{deg} G+\operatorname{deg} Q)=\operatorname{deg} G+\operatorname{deg} Q .
$$

According to the calculations preceding (2.3) we have

$$
\operatorname{deg} G=\sum_{k=1}^{\max |J|} \varphi(k) \cdot \max _{\mathbf{b} \in \mathscr{E}} \max \left(0, \chi_{k}(\mathbf{b})\right),
$$

and thus

$$
\operatorname{deg}(F-G \cdot Q) \leq \sum_{k=1} \varphi(k) \cdot \max _{\mathbf{b} \in \mathscr{E}} \max \left(0, \chi_{k}(\mathbf{b})\right)+\operatorname{deg} Q<\varphi(m)
$$

in view of the assumptions of the lemma. Therefore $F-G \cdot Q=0$ in $\mathbf{Q}[\Lambda]$.

Applying Lemma 2.8 we conclude that $Q$ has to be constant, and by Corollary 3.2(a) $P-Q \in D_{\mathbf{Q}}(J)$.

Corollary 3.4. Let $P$ and $Q$ be as in Lemma 3.3. If $\operatorname{deg} Q \geq 1$ then

$$
\sum_{k=1}^{\max |J|} \varphi(k) \cdot \max _{\mathbf{b} \in \mathscr{C}} \max \left(0, \chi_{k}(\mathbf{b})\right) \geq \varphi(m)-\operatorname{deg} Q,
$$

and hence

$$
\sum_{j \in|J|} j \cdot \operatorname{deg}_{ \pm j}(P) \geq \varphi(m)-\operatorname{deg} Q .
$$

\section{CONCENTRATED CYCLIC ACTIONS AND INTEGRALITY RESUlTS}

In this section we shall apply the results of the two preceding sections to the $g$-signature formula (1.4). It will be assumed throughout that $J \subseteq(-m / 2, m / 2]$ and, moreover, that for any $\sigma \in(\mathbf{Z} / m \mathbf{Z})^{*}$ the set $\sigma J$ is reduced modulo $m$ so that $\sigma J \subseteq(-m / 2, m / 2]$.

Given a $g$-representation $g \rightarrow \psi(g), \psi^{\sigma}$ denotes the $g$-representation $g \rightarrow$ $\psi\left(g^{\sigma}\right)$.

Definition 4.1. A diffeomorphism $g: M \rightarrow M$ of period $m$, satisfying the assumptions of Theorem $\mathrm{A}$, is

(1) weakly $\sigma$-concentrated-if the polynomial $\mathscr{N}_{\left(M, g^{\sigma}\right)} \in \mathbf{Z}\left[\left\{X_{\sigma j}\right\}_{j \in J}\right]$ satisfies $\delta_{\sigma J}\left(\mathscr{N}_{\left(M, g^{\sigma}\right)}\right)<\varphi(m)$;

(2) $\sigma$-concentrated -if the generic polynomial

$$
P=\sum_{\psi} \sum_{(\mathbf{b}, \mathbf{c}) \in \mathcal{O}_{\psi}{ }^{\sigma}} a_{\mathbf{b}, \mathbf{c}} \prod_{j \in\left[\psi^{\sigma}\right]} X_{j}^{b_{j}}\left(X_{j}^{2}-1\right)^{c_{j}}
$$


(where $a_{\mathbf{b}, \mathbf{c}} \in \mathbf{Q}$ and $\mathscr{O}_{\psi^{\sigma}}$ is defined as in (1.3)) has the property $\delta_{\sigma J}(P)<$ $\varphi(m)$.

Note that the property of an action being weakly $\sigma$-concentrated does not depend on $\mathscr{N}_{\left(M, g^{\sigma}\right)}$ but only on its residue class modulo $D_{\mathbf{Q}}(\sigma J)$.

Let $g: M \rightarrow M$ be as in Theorem A. Write

$$
\operatorname{sign}(g, M)=\sum_{j=0}^{\mu} s_{j} \lambda_{1 / m}^{j} \in \mathbf{Z}\left[\lambda_{1 / m}\right],
$$

where each $s_{j}$ is an integer, $\mu<\varphi(m)$, and $s_{\mu} \neq 0$ (if $\operatorname{Sign}(g, M)=0$, then $\mu=0)$.

Definition 4.2. A diffeomorphism $g: M \rightarrow M$ is strongly $\sigma$-concentrated if the generic $P$ given in (4.1) has the property $\operatorname{deg} G<\varphi(m)-\mu$, where $P\left(\left\{\left(\Lambda^{j}+1\right) /\left(\Lambda^{j}-1\right)\right\}_{j \in \sigma J}\right)=F(\Lambda) / G(\Lambda), F$ and $G$ being relatively prime polynomials.

Remark 4.3. Since $\operatorname{deg} F \leq \operatorname{deg} G$, being strongly $\sigma$-concentrated implies being $\sigma$-concentrated, which in turn obviously implies being weakly $\sigma$-concentrated.

Remark 4.4. Definition 4.1(2) relies solely on the combinatorial information provided by $\operatorname{dim} M$ and the $\psi$ 's; Definition 4.2 requires, in addition, a knowledge of $\operatorname{Sign}(g, M)$.

Denote $\sigma \mathscr{O}=\bigcup_{\psi} \mathscr{O}_{\psi^{\sigma}}$. Employing the function $\tilde{\chi}_{k}: \mathbf{Z}_{+}^{\sigma J} \times \mathbf{Z}_{+}^{\sigma J} \rightarrow \mathbf{Z}$ defined in (2.4) we can obtain alternatives to Definitions 4.1(2) and 4.2.

Lemma 4.5. Let $g: M \rightarrow M$ be as in Theorem A. Then $g$ is

(i) $\sigma$-concentrated iff

$$
\sum_{k=1}^{\max |\sigma J|} \varphi(k) \cdot \max _{(\mathbf{b}, \mathbf{c} \in \in \sigma} \max \left(0, \tilde{\chi}_{k}(\mathbf{b}, \mathbf{c})\right)<\varphi(m)+\varepsilon \min |\sigma J|,
$$

where $\varepsilon=1$ if all the $d_{\psi}$ 's are odd and 0 otherwise;

(ii) strongly $\sigma$-concentrated iff

$$
\sum_{k=1}^{\max |\sigma J|} \varphi(k) \cdot \max _{(\mathbf{b}, \mathbf{c} \in \in \sigma \Theta} \max \left(0, \tilde{\chi}_{k}(\mathbf{b}, \mathbf{c})\right)<\varphi(m)-\mu .
$$

Proof. (i) Recall that, according to the discussion preceding Lemma 2.9, the degree of $F(\Lambda)$ for a generic polynomial $P$ of the form given in (4.1) differs from the left-hand side of (4.2) by $\min _{c} \sum_{j \in \sigma J}|j| c_{j}$. Thus it remains to show that $\min _{\mathrm{c}} \sum_{j \in \sigma J}|j| c_{j}=\varepsilon \min |\sigma J|$, which follows routinely from (1.3).

(ii) This part is an immediate implication of the fact that the left-hand side of (4.3) is, for a generic $P$, the degree of $G(\Lambda)$.

One can provide sufficient conditions for a diffeomorphism to be (strongly) $\sigma$-concentrated which are more geometric and easier to verify than those of Lemma 4.5. 
Put $n_{ \pm j}\left(\psi^{\sigma}\right)=n_{j}\left(\psi^{\sigma}\right)+n_{-j}\left(\psi^{\sigma}\right)$ for $j \neq m / 2$ and $n_{ \pm m / 2}\left(\psi^{\sigma}\right)=n_{m / 2}\left(\psi^{\sigma}\right)$.

Lemma 4.6. Each of the following conditions implies its successor:

(i) $\left(\sum_{j \in|\sigma J|} j\right) \cdot \operatorname{dim} M / 2<\varphi(m)$.

(ii) $\sum_{j \in|\sigma J|} j \cdot \max _{\left\{\psi^{\sigma}\right\}}\left[n_{ \pm j}\left(\psi^{\sigma}\right)+\operatorname{dim} M_{\psi^{\sigma}}^{g} / 2\right]<\varphi(m)$.

(iii) $g: M \rightarrow M$ is $\sigma$-concentrated.

(iv) $g: M \rightarrow M$ is weakly $\sigma$-concentrated.

Proof. The implication (i) $\Rightarrow$ (ii) is trivial, while (iii) $\Rightarrow$ (iv) is the contents of Remark 4.3. To prove the implication (ii) $\Rightarrow$ (iii), we just note that for any $P$ we have $\delta_{\sigma J}(P) \leq \sum_{j \in|\sigma J|} j \cdot \operatorname{deg}_{ \pm j} P$, and for a generic $P$ as in (4.1)

$$
\operatorname{deg}_{ \pm j} P \leq \max _{\left\{\psi^{\sigma}\right\}}\left(n_{ \pm j}\left(\psi^{\sigma}\right)+\operatorname{dim} M_{\psi^{\sigma}}^{g} / 2\right) .
$$

Similarly we have

Lemma 4.7. Each of the following conditions implies its successor:

(i) $\left(\sum_{j \in|\sigma J|} j\right) \cdot \operatorname{dim} M / 2<\varphi(m)-\mu$.

(ii) $\sum_{j \in|\sigma J|} j \cdot \max _{\left\{\psi^{\sigma}\right\}}\left[n_{ \pm j}\left(\psi^{\sigma}\right)+\operatorname{dim} M_{\psi^{\sigma}}^{g} / 2\right]<\varphi(m)-\mu$.

(iii) $g: M \rightarrow M$ is strongly $\sigma$-concentrated.

Our next objective is to clarify to what extent the invariant $\operatorname{Sign}(g, M)$ and the list of normal representations $\{\psi\}$ determine the characteristic numbers $a_{\mathbf{b}, \mathbf{c}}\left(\nu_{\psi}\right),(\mathbf{b}, \mathbf{c}) \in \mathscr{O}_{\psi}$, for $\sigma$-concentrated actions.

Proposition 4.8. Suppose that the orientation-preserving diffeomorphisms $g: M_{1}$ $\rightarrow M_{1}$ and $g: M_{2} \rightarrow M_{2}$ of period $m$ possess the following properties:

(i) $\operatorname{dim} M_{1}=\operatorname{dim} M_{2}$.

(ii) The normal bundles $\nu\left(M_{1}^{g}, M_{1}\right)$ and $\nu\left(M_{2}^{g}, M_{2}\right)$ both admit $g$-equivariant complex structures.

(iii) The list of (complex) slice-types $\{\psi\}$ of points of $M_{1}^{g}$ coincides with that of $M_{2}^{g}$.

(iv) $\operatorname{Sign}\left(g, M_{1}\right)=\operatorname{Sign}\left(g, M_{2}\right)$.

(v) One of the actions (and thus both) is $\sigma$-concentrated for some $\sigma \in$ $(\mathbf{Z} / m \mathbf{Z})^{*}$.

Then the polynomial $\mathscr{N}_{\left(M_{1} \cup-M_{2}, g^{\sigma}\right)}$, where $\sigma J=\bigcup_{\psi}\left[\psi^{\sigma}\right]$, is $\sigma J$-diagonal. Proof. Due to (iii) and (iv), Theorem A implies

$$
\mathcal{N}_{\left(M_{1} \cup-M_{2}, g\right)}=\mathcal{N}_{\left(M_{1}, g\right)}-\mathcal{N}_{\left(M_{2}, g\right)} \in \operatorname{Ker} \nabla_{J}
$$

(see $\S 3$ for the definition of $\left.\nabla_{J}\right)$. Employing Galois $(\mathbf{Z} / m \mathbf{Z})^{*}$-symmetry,

$$
\mathscr{N}_{\left(M_{1} \cup-M_{2}, g^{\sigma}\right)} \in \operatorname{Ker} \nabla_{\sigma J} \text {. }
$$

From (v) and Remark 4.4 it follows that the action $g: M_{1} \cup-M_{2} \rightarrow M_{1} \cup-M_{2}$ is $\sigma$-concentrated. By Corollary 3.2(b) and in view of Lemma 4.5, $\mathscr{N}_{\left(M_{1} \cup-M_{2}, g^{\sigma}\right)}$ is $\sigma J$-diagonal. 
Applying Lemma 2.5 to $\mathscr{N}_{\left(M_{1} \cup-M_{2}, g^{\sigma}\right)}$ we obtain

Corollary 4.9. Let $g: M \rightarrow M$ be as in Theorem A and $\sigma$-concentrated. Then for any $l \in \mathbf{N}$ such that $l \nmid j, j \in \sigma J$, and any $r$ relatively prime to $l$, the value of the polynomial $\mathscr{N}_{\left(M, g^{\sigma}\right)}$ at the point $\left\{\alpha_{j r / l}\right\}_{j \in \sigma J}$, i.e.,

$$
\sum_{\psi} \sum_{\omega \in \Omega\left(\psi^{\sigma}\right)}(-1)^{d_{\psi}}\left[\prod_{j \in\left[\psi^{\sigma}\right]} \alpha_{j r / l}^{n_{j}\left(\psi^{\sigma}\right)+\left\|_{j} \omega\right\|-\left.2\right|_{j} \omega \mid}\left(\alpha_{j r / l}^{2}-1\right)^{|, \omega|}\right] \cdot \mathscr{S}_{\omega}\left(\nu_{\psi^{\sigma}}\right),
$$

is completely determined by $\operatorname{Sign}(g, M)$, provided that $\operatorname{dim} M$ and the $\psi$ 's are fixed.

In particular, under the previous assumptions this value, lying in $\mathbf{Q}\left(\lambda_{1 / l}\right)$, is an invariant of the g-homology type of $M$ (in the category of $\sigma$-concentrated actions).

Later (see Theorems 4.24-4.25) we shall provide a more detailed formula computing $\mathscr{N}_{\left(M, g^{\sigma}\right)}\left(\left\{\alpha_{j / l}\right\}_{j \in \sigma J}\right)$ for $2 \leq l \leq 4$.

Lemma 2.10 now yields

Corollary 4.10. Let $g: M \rightarrow M$ be as in Theorem A and $\sigma$-concentrated. Then for any $l \in \mathbf{N}$ the value of the $\operatorname{deg}_{\mathbf{t}_{l}}$-homogeneous part ${ }_{\mathbf{t}_{l}} \mathcal{N}_{\left(M, g^{\sigma}\right)}$ of the polynomial $\mathscr{N}_{\left(M, g^{\sigma}\right)}$ at the point $\gamma_{l}$ given by

$$
\gamma_{l, j}= \begin{cases}j^{(-1)^{1+2 j / l},}, & l \mid 2 j, \\ \alpha_{j / l}, & l \nmid 2 j,\end{cases}
$$

is completely determined by $\operatorname{Sign}(g, M)$, and is thus an invariant of the $g$ homology type of $M$ (within the category of $\sigma$-concentrated actions with the prescribed $\operatorname{dim} M$ and $\psi$ 's).

Given a periodic diffeomorphism $g: M \rightarrow M$ as in Theorem A, define a function $\mathscr{A}_{(M, g)}: \mathscr{O} \rightarrow \mathbf{Z}$, where $\mathscr{O}=\bigcup_{\psi} \mathscr{O}_{\psi} \subseteq \mathbf{Z}_{+}^{J} \times \mathbf{Z}_{+}^{J}$, by

$$
\mathscr{A}_{(M, g)}(\mathbf{b}, \mathbf{c})=\sum_{\left\{\psi:(\mathbf{b}, \mathbf{c}) \in \mathbb{Q}_{\psi}\right\}} a_{\mathbf{b}, \mathbf{c}}\left(\nu_{\psi}\right) \text {. }
$$

(This is the coefficient of $\prod_{j \in J} X_{j}^{b_{j}}\left(X_{j}^{2}-1\right)^{c_{j}}$ in the formula defining the polynomial $\mathscr{N}_{(M, g)}$.) Obviously, if $\operatorname{dim} M_{1}=\operatorname{dim} M_{2}$ and $\left(M_{1}, g\right),\left(M_{2}, g\right)$ have the same list of $\psi$ 's, then $\mathscr{A}_{\left(M_{1} \cup-M_{2}, g\right)}=\mathscr{A}_{\left(M_{1}, g\right)}-\mathscr{A}_{\left(M_{2}, g\right)}$. If, in addition, $g: M_{1} \rightarrow M_{1}$ is $\sigma$-concentrated and $\operatorname{Sign}\left(g, M_{1}\right)=\operatorname{Sign}\left(g, M_{2}\right)$, then, by Proposition 4.9, $\mathscr{N}_{\left(M_{1} \cup-M_{2}, g^{\sigma}\right)}$ is $\sigma J$-diagonal. In view of Lemma 3.1 and Corollary 3.2, putting

$$
T=\sum_{k=1}^{\max |\sigma J|} \varphi(k) \max _{(\mathbf{b}, \mathbf{c}) \in \mathscr{\theta}} \max \left(0, \tilde{\chi}_{k}\left(\sigma^{-1} \mathbf{b}, \sigma^{-1} \mathbf{c}\right)\right)-\varepsilon \cdot \min |\sigma J|,
$$

this is equivalent to

$$
\mathcal{N}_{\left(M_{1} \cup-M_{2}, g^{\sigma}\right)}\left(\left\{\left(l^{j}+1\right) /\left(l^{j}-1\right)\right\}_{j \in \sigma J}\right)=0, \quad 2 \leq l \leq T+2 .
$$

This imposes a system of linear constraints on the numbers $\mathscr{A}_{\left(M_{1} \cup-M_{2}, g^{\sigma}\right)}(\mathbf{b}, \mathbf{c})$, 
where $(\mathbf{b}, \mathbf{c})$ runs over $\sigma \mathscr{O}$. This yields

Theorem 4.11. Consider the category of $\sigma$-concentrated orientation-preserving diffeomorphisms $g: M \rightarrow M$ of period $m\left(\sigma \in(\mathbf{Z} / m \mathbf{Z})^{*}\right)$ with prescribed $\operatorname{dim} M, \operatorname{Sign}(g, M)$, and list of (complex) slice-types $\psi$ for the points of $M^{g}$, such that the normal bundle $\nu\left(M^{g}, M\right)$ admits a g-equivariant complex structure.

Let $\mathscr{A}_{\left(M, g^{\sigma}\right)}: \sigma \mathscr{O} \rightarrow \mathbf{Z}$ be as in (4.4) and define $\theta_{l}: \sigma \mathscr{O} \rightarrow \mathbf{Q}, l \geq 2$, by

$$
\theta_{l}(\mathbf{b}, \mathbf{c})=\prod_{j \in \sigma J}\left(4 l^{j}\right)^{c_{j}}\left(l^{j}+1\right)^{b_{j}} /\left(l^{j}-1\right)^{b_{j}+2 c_{j}} .
$$

Then the dimension of the affine subspace spanned by the $\mathscr{A}_{\left(M, g^{\sigma}\right)}$ 's satisfies

$$
\operatorname{dim}_{\mathbf{Q}}\left(\operatorname{Span}\left\{\mathscr{A}_{\left(M, g^{\sigma}\right)}\right\}\right) \leq \#(\mathscr{O})-\operatorname{dim}_{\mathbf{Q}}\left(\operatorname{Span}\left\{\theta_{l}\right\}_{2 \leq l \leq T+2}\right), \quad T \in \mathbf{N} .
$$

(By Corollary 3.2 the right-hand side actually stabilizes when $T$ is as in (4.5).)

In particular, if the functions $\left\{\theta_{l}\right\}, 2 \leq l \leq T+2$, separate the points of $\mathscr{O}$, the characteristic numbers $\mathscr{A}_{\left(M, g^{\sigma}\right)}(\mathbf{b}, \mathbf{c}),(\mathbf{b}, \mathbf{c}) \in \mathscr{O}$, (which are special combinations of the normal quasi-signatures), are uniquely determined.

We shall now consider a special case in which the polynomial $\mathscr{N}_{(M, g)}$ is easier to handle.

Proposition 4.12. Let $g: M \rightarrow M$ belong to the category introduced in Theorem 4.11. Assume that for each $\psi$, the set $[\psi]$ is a singleton (i.e., each $\psi(g)$ is a scalar matrix) and that $\psi_{1} \neq \psi_{2}$ implies $\left\{|j|: j \in\left[\psi_{1}\right]\right\} \neq\left\{|j|: j \in\left[\psi_{2}\right]\right\}$. Denote

$$
\Omega\left(\psi, d_{\psi}, k\right)=\left\{\omega \in \Omega\left(\psi ; d_{\psi}\right): n_{\psi}+\|\omega\|-2|\omega| \leq k \leq n_{\psi}+\|\omega\|\right\},
$$
$k \in \mathbf{Z}_{+}, n_{\psi}=\operatorname{dim}_{\mathbf{C}} \psi$. Then for any $\psi$ and $k \in \mathbf{Z}$, the characteristic number

$$
\sum_{\omega \in \Omega\left(\psi ; d_{\psi}, k\right)}(-1)^{\left(\|\omega\|-n_{\psi}-k\right) / 2}\left(\begin{array}{c}
|\omega| \\
\left(\|\omega\|+n_{\psi}-k\right) / 2
\end{array}\right) \mathscr{S}_{\omega}\left(\nu_{\psi}\right)
$$

is uniquely determined, and is thus an invariant of the g-homology type of $M$. This invariant vanishes if $\operatorname{Sign}(g, M)$ does. Moreover, for any $k \leq n_{\psi}$, the analogous conclusions hold for the characteristic number

$$
\sum_{\left\{c:(k, c) \in \mathcal{O}_{\psi}\right\}}(-1)^{c} a_{\mathbf{k}, \mathbf{c}}\left(\nu_{\psi}\right) .
$$

Prior to proving the proposition, we shall present several implications.

Corollary 4.13. Let $g: M^{2 d} \rightarrow M^{2 d}$ be an orientation-preserving diffeomorphism of period $m$ such that $\nu\left(M^{g}, M\right)$ admits a complex structure. Assume that $M^{g}$ is of real codimension 2 in $M$ and that for some $\sigma \in(\mathbf{Z} / m \mathbf{Z})^{*}$

$$
d \cdot \sum_{\{k>0: k \mathbf{Z} \cap \sigma J \neq \varnothing\}} \varphi(k)+\delta \cdot \sum_{\{k>0:(k / 2)(2 \mathbf{Z}+1) \cap \sigma J \neq \varnothing\}} \varphi(k)<\varphi(m)+\varepsilon \min |\sigma J|,
$$


where

$$
(\delta, \varepsilon)= \begin{cases}(0,0), & d=1, \\ (1,0), & d \geq 3, d \equiv 1(\bmod 2) \\ (2,1), & d \equiv 0(\bmod 2)\end{cases}
$$

Then the invariant

$$
\operatorname{Sign}\left(\pitchfork^{k+1} M_{\psi}^{g}\right)+(-1)^{d} \operatorname{sign}\left(\pitchfork^{k+1} M_{\bar{\psi}}^{g}\right)
$$

(where $\bar{\psi}$ is the complex conjugate of $\psi$ ) is uniquely determined by $\operatorname{Sign}(g, M)$ for any slice-type $\psi$ and $k \in \mathbf{Z}$. It vanishes when $\operatorname{Sign}(g, M)$ does.

Proof. Since $\operatorname{codim}\left(M^{g}, M\right)=2$, for each $\psi$ the set $[\psi]$ is a singleton. $\operatorname{Re}$ placing $X_{[\bar{\psi}]}$ by $-X_{[\psi]}$ in $\mathscr{N}_{(M, g)}$ if necessary, it follows that our action is of the type described in Proposition 4.12. Applying that proposition with $n_{\psi}=1$ consecutively for $k$ 's descending from $d$ to 0 , we see that the numbers

$$
\mathscr{S}_{\omega}\left(\nu_{\psi}\right)+(-1)^{d} \mathscr{S}_{\omega}\left(\nu_{\bar{\psi}}\right)=\operatorname{Sign}\left(\pitchfork^{\omega+1} M_{\psi}^{g}\right)+(-1)^{d} \operatorname{Sign}\left(\pitchfork^{\omega+1} M_{\bar{\psi}}^{g}\right)
$$

are uniquely determined by $\operatorname{Sign}(g, M)$. It remains to verify that condition (i) of Lemma 4.5, ensuring that an action is $\sigma$-concentrated, reduces in our case to the inequality given in the corollary, which is routine. This proves the corollary.

Note that, by Lemma 4.6 , the inequality $\left(\sum_{j \in|\sigma J|} j\right) d<\varphi(m)$ implies the one in Corollary 4.13.

Example 4.14. (a) Let $g: M^{2} \rightarrow M^{2}$ be an orientation-preserving diffeomorphism of period $m$ of a surface $M^{2}$. If $\sum_{\{k>0: k \mathbf{Z} \cap \sigma J \neq \varnothing\}} \varphi(k)<\varphi(m)$ for some $\sigma \in(\mathbf{Z} / m \mathbf{Z})^{*}$, then, for each $\psi, \#\left(M_{\psi}^{g}\right)-\#\left(M_{\frac{g}{\psi}}^{g}\right)$ is uniquely determined by $\operatorname{Sign}(g, M)$ and vanishes when the latter does. Thus, if $\operatorname{Sign}(g, M)=0$, then, by Corollary $2.11, M$ is an equivariant sum of several copies of $S_{\psi}^{2}$ and a surface $M^{\prime}$ carrying a $g$-fixed point free action. The action on each sphere $S_{\psi}^{2}$ is a rotation by the angle $2 \pi[\psi] / m$.

(b) Let $g: M^{4} \rightarrow M^{4}$ be an orientation-preserving diffeomorphism as in Corollary 4.13. If for some $\sigma \in(\mathbf{Z} / m \mathbf{Z})^{*}$ we have $2 \sum_{j \in|\sigma J|} j<\varphi(m)$, then, for each $\psi$, the self-intersection number $\#\left(M_{\psi}^{g} \pitchfork M_{\psi}^{g}\right)+\#\left(M_{\bar{\psi}}^{g} \pitchfork M_{\frac{g}{\psi}}^{g}\right)$ is uniquely determined by $\operatorname{Sign}(g, M)$ and vanishes when the latter does. Thus if such a $\sigma$-concentrated action is complex algebraic, then $\operatorname{Sign}(g, M)=0$ implies that the bundles $\nu\left(M_{\psi}^{g}, M\right)$ are trivial. In general, the nonnegativity of $\#\left(M_{\psi}^{g} \pitchfork M_{\psi}^{g}\right)$ for algebraic actions seems to put some constraints on the possible values of $\operatorname{Sign}(g, M)$.

Corollary 4.15. Let $g: M^{2 d} \rightarrow M^{2 d}$ be as in Proposition 4.12. Then for each $\psi$ :

(i) If $d$ is even, then $\operatorname{Sign}\left(M_{\psi}^{g} \pitchfork M_{\psi}^{g}\right)$ is uniquely determined by $\operatorname{Sign}(g, M)$ and vanishes when the latter does. 
(ii) If $d$ is odd, then $\mathscr{S}_{(1,1, \ldots, 0)}\left(\nu_{\psi}\right)-\mathscr{S}_{(2,1, \ldots, 1)}\left(\nu_{\psi}\right)$ is uniquely determined by $\operatorname{Sign}(g, M)$ and vanishes when the latter does.

(iii) $\left.\sum_{\left\{\omega \in \Omega\left(\psi, d_{\psi}\right)\right.}:\|\omega\|=d_{\psi}\right\} \mathscr{S}_{\omega}\left(\nu_{\psi}\right)$ is uniquely determined by $\operatorname{Sign}(g, M)$ and vanishes when the latter does.

In fact, parts (i), (ii), (iii) follow straightforwardly from Proposition 4.12 choosing $k=0,1, d$, respectively. In each case we have to describe explicitly the set $\Omega\left(\psi, d_{\psi}, k\right)$, and for (i) we also note that, by $(1.2), \mathscr{S}_{(1,1, \ldots, 1)}\left(\nu_{\psi}\right)=$ $\operatorname{Sign}\left(M_{\psi}^{g} \pitchfork M_{\psi}^{g}\right)$.

Proof of Proposition 4.12. As mentioned already, the polynomial $\mathscr{N}_{\left(M^{\prime}, g^{\sigma}\right)}-$ $\mathcal{N}_{\left(M^{\prime \prime}, g^{\sigma}\right)}$ is $\sigma J$-diagonal for any two actions $g: M^{\prime} \rightarrow M^{\prime}, g: M^{\prime \prime} \rightarrow M^{\prime \prime}$ in our category. Since the $[\psi]$ 's are singletons, this polynomial is of the form $\sum_{j \in \sigma J} P_{j}\left(X_{j}\right)$ (we identify a representation $\psi$ with the unique $j \in[\psi]$ ). According to Corollary 2.11, each $P_{j}\left(X_{j}\right)+P_{-j}\left(X_{-j}\right)$ is a constant. (According to our hypothesis, $j \in \sigma J$ implies $-j \notin \sigma J$.) In view of Theorem A

$$
P_{j}\left(X_{j}\right)=\sum_{\omega \in \Omega\left(\psi ; d_{\psi}\right)}(-1)^{d_{\psi}} X_{j}^{n_{j}+\|\omega\|-2|\omega|}\left(X_{j}^{2}-1\right)^{|\omega|}\left[\mathscr{S}_{\omega}\left(\nu_{\psi}^{\prime}\right)-\mathscr{S}_{\omega}\left(\nu_{\psi}^{\prime \prime}\right)\right]
$$

for each $\psi$. Now if such a polynomial is a constant, then it is actually 0 . Writing down explicitly the list of equalities this yields, we get the proposition.

We shall next start considering strongly $\sigma$-concentrated actions.

Proposition 4.16. If $g: M \rightarrow M$ is strongly $\sigma$-concentrated, then $\operatorname{Sign}(g, M) \in$ Z. In particular, if $\operatorname{dim} \equiv 2(\bmod 4)$, then $\operatorname{Sign}(g, M)$ must vanish.

Proof. Write $\operatorname{Sign}\left(g^{\sigma}, M\right)=Q\left(\lambda_{1 / m}\right)$ for an appropriate $Q \in \mathbf{Z}[\Lambda]$ with $\mu=$ $\operatorname{deg} Q<\varphi(m)$. Since $\mathcal{N}_{\left(M, g^{\sigma}\right)}\left(\left\{\alpha_{j / m}\right\}_{j \in \sigma J}\right)=\operatorname{Sign}\left(g^{\sigma}, M\right)$, and the action is strongly $\sigma$-concentrated, the assumptions of Lemma 3.3 are fulfilled with $P=\mathscr{N}_{\left(M, g^{\sigma}\right)}$. Consequently $\mu=0$, which means that $\operatorname{Sign}\left(g^{\sigma}, M\right) \in \mathbf{Z}$, so that $\operatorname{Sign}(g, M) \in \mathbf{Z}$. This proves the proposition.

Lemma 4.5 now yields

Corollary 4.17. The following conditions are equivalent, provided not all $d_{\psi}$ 's are odd:

(i) The action is strongly $\sigma$-concentrated.

(ii) The action is $\sigma$-concentrated and $\operatorname{Sign}(g, M) \in \mathbf{Z}$.

Corollary 4.18. Let $g: M \rightarrow M$ be as in Theorem A. Suppose $\operatorname{Sign}(g, M) \notin \mathbf{Z}$. Write $\operatorname{Sign}(g, M)=\sum_{j=0}^{\mu} s_{j} \lambda_{1 / m}^{j}$ for suitable integers $s_{j}$. Then the following inequalities (each implying its successor) hold:
(i) $\sum_{k=1}^{\max |J|} \varphi(k) \cdot \max _{(\mathbf{b}, \mathbf{c}) \in \mathcal{O}} \max \left(0, \tilde{\chi}_{k}(\mathbf{b}, \mathbf{c})\right) \geq \varphi(m)-\mu$.
(ii) $\sum_{j \in|J|} j \cdot \max _{\psi}\left(n_{ \pm j}(\psi)+d_{\psi}\right) \geq \varphi(m)-\mu$.
(iii) $\sum_{j \in|J|} j \geq 2(\varphi(m)-\mu) / \operatorname{dim} M$. 
In view of Lemma 4.7, the corollary follows by negation from Proposition 4.16 and Corollary 3.4.

Remark 4.19. Roughly speaking, the corollary states that, if $\operatorname{Sign}(g, M)$ is "concentrated", i.e., $\mu$ is "not too close" to $\varphi(m)$ relatively to $\operatorname{dim} M$ (but is still nonzero), then the normal eigenvalues $\left\{\lambda_{1 / m}^{j}\right\}_{j \in J}$ of the action are "dispersed". For example, if $g: M^{2} \rightarrow M^{2}$ is an orientation-preserving diffeomorphism with $\operatorname{Sign}(g, M) \neq 0$, then the normal eigenvalues are such that

$$
\sum_{\{k>0: k \mathbf{Z} \cap J \neq \varnothing\}} \varphi(k) \geq \varphi(m)-\mu .
$$

It was shown that, given $\sigma$-concentrated action $g: M \rightarrow M$ with $\operatorname{Sign}(g, M)$ $\in \mathbf{Z}$, the polynomial $\mathscr{N}_{\left(M, g^{\sigma}\right)}-\operatorname{Sign}\left(g^{\sigma}, M\right)$ is $\sigma J$-diagonal. It therefore vanishes under the substitutions discussed in Corollary 2.4. Substituting $\left\{X_{j}=\Delta(j)\right\}_{j \in \sigma J}$ we realize that the only nonvanishing terms correspond to all $\omega$ 's being 0 's, for which $\mathscr{S}_{\omega}\left(\nu_{\psi}\right)=\operatorname{Sign}\left(M_{\psi}^{g}\right)$. Note that

$$
\prod_{j \in\left[\psi^{\sigma}\right]} \Delta(j)^{n_{j}\left(\psi^{\sigma}\right)}=(-1)^{\varepsilon\left(\psi^{\sigma}\right)}, \quad \varepsilon\left(\psi^{\sigma}\right)=\sum_{j<0: j \in\left[\psi^{\sigma}\right]} n_{j}\left(\psi^{\sigma}\right)
$$

This yields

Theorem 4.20. Let $g: M \rightarrow M$ be an orientation-preserving diffeomorphism of period $m$ such that $\nu\left(M^{g}, M\right)$ admits a g-equivariant complex structure. Assume that $\operatorname{Sign}(g, M) \in \mathbf{Z}$ and the action is $\sigma$-concentrated (see Definition 4.1) for some $\sigma \in(\mathbf{Z} / m \mathbf{Z})^{*}$. Then, employing the complex structure in $\nu\left(M^{g}, M\right)$ to orient the $M_{\psi}^{g}$, s, we have

$$
\operatorname{Sign}(g, M)=\sum_{\psi}(-1)^{\varepsilon\left(\psi^{\sigma}\right)} \operatorname{Sign}\left(M_{\psi}^{g}\right)
$$

where $\varepsilon\left(\psi^{\sigma}\right)=\sum_{j<0: j \in\left[\psi^{\sigma}\right]} n_{j}\left(\psi^{\sigma}\right)$.

This result should be compared with [BK, Theorem 7.8] which is sort of a modulo 4 version thereof.

Similarly, we can employ the substitutions from Lemma 2.5. Substituting $\left\{X_{j}=0\right\}_{j \in \sigma J}$ in $\mathscr{N}_{\left(M, g^{\sigma}\right)}-\operatorname{Sign}\left(g^{\sigma}, M\right)$ in accordance with part (1) of that lemma, we observe that the only survivors are the terms with $\omega$ 's satisfying $n_{j}\left(\psi^{\sigma}\right)+\left\|{ }_{j} \omega\right\|-\left.2\right|_{j} \omega \mid=0$ for each $\psi$ and $j \in\left[\psi^{\sigma}\right]$, namely only the $\omega$ 's of the form $(1,1, \ldots, 1)$. As noted in $\S 1, \mathscr{S}_{(1,1, \ldots, 1)}\left(\nu_{\psi}\right)=\operatorname{Sign}\left(M_{\psi}^{g} \pitchfork M_{\psi}^{g}\right)$. 
Thus, we have proved

Theorem 4.21. Let $g: M \rightarrow M$ be as in Theorem 4.20. Assume that each $j \in \sigma J$ is odd. Then under an appropriate choice of orientations

$$
\operatorname{Sign}(g, M)=\sum_{\psi} \operatorname{Sign}\left(M_{\psi}^{g} \pitchfork M_{\psi}^{g}\right) .
$$

Now we shall use the substitutions corresponding to $l=3$ and $l=4$ in Lemma 2.5. Some notations are required first. Denote by $\omega^{+}$(resp. $\omega^{-}$) the part of $\omega$ consisting of those rows enumerated by $j$ 's satisfying $j \equiv 1(\bmod l)$ $($ resp. $j \equiv-1(\bmod l))$. The vectors $\mathbf{n}^{+}(\psi)$ and $\mathbf{n}^{-}(\psi)$ are similarly defined, and for $l=4$, let $\mathbf{n}^{(2)}(\psi)$ be the projection of $\mathbf{n}(\psi)$ on the subspace corresponding to those $j$ 's with $j \equiv 2(\bmod 4)$. Set $\delta(\mathbf{n}(\psi), \omega)=$ $\left\|\mathbf{n}^{+}(\psi)\right\|-\left\|\mathbf{n}^{-}(\psi)\right\|-\left\|\omega^{+}\right\|+\left\|\omega^{-}\right\|, \rho(d)=\left((-1)^{d}-1\right) / 2$, and $\varepsilon(d, \mathbf{n}(\psi), \omega)=$ $(\delta(\mathbf{n}(\psi), \omega)+\rho(d)) / 2$. For $l=4$ we also consider the set $\Omega^{(2)}(\psi)$ given by

$$
\Omega^{(2)}(\psi)=\left\{\omega \in \Omega(\psi): \omega_{j, k}=1, j \equiv 2(4), 1 \leq k \leq n_{j}(\psi)\right\} .
$$

Routine calculations, employing the substitutions (2) and (3) of Lemma 2.5 for the $\sigma J$-diagonal polynomial $\mathscr{N}_{\left(M, g^{\sigma}\right)}-\operatorname{Sign}\left(g^{\sigma}, M\right)$, lead to

Theorem 4.22. Let $g: M^{2 d} \rightarrow M^{2 d}$ be as in Theorem 4.20.

(i) If $3 \nmid j \quad j \in \sigma J$, then

$\operatorname{Sign}(g, M)=\sum_{\psi} \sum_{\omega \in \Omega\left(\psi^{\sigma}\right)}(-1)^{\varepsilon\left(d, \mathbf{n}\left(\psi^{\sigma}\right), \omega\right)} \cdot(1 / 3)^{\left(\left\|\mathbf{n}\left(\psi^{\sigma}\right)\right\|+\|\omega\|+\rho(d)\right) / 2} \cdot 4^{|\omega|} \mathscr{S}_{\omega}\left(\nu_{\psi^{\sigma}}\right)$.

(ii) If $4 \nmid j, j \in \sigma J$, then

$$
\operatorname{Sign}(g, M)=\sum_{\psi} \sum_{\omega \in \Omega\left(\psi^{\sigma}\right)}(-1)^{\varepsilon\left(d, \mathbf{n}\left(\psi^{\sigma}\right), \omega\right)} \cdot 2^{|\omega|-\left|\mathbf{n}^{(2)}\left(\psi^{\sigma}\right)\right|} \mathscr{S}_{\omega}\left(\nu_{\psi^{\sigma}}\right)
$$

(For odd $d, \operatorname{Sign}(g, M)$ has to vanish.)

We shall derive several consequences of the preceding theorems.

Corollary 4.23. Let $g: M \rightarrow M$ be a $\sigma$-concentrated action with $\operatorname{Sign}(g, M) \in$ $\mathrm{Z}$. Assume that all $j \in \sigma J$ are odd. If the Euler class of each bundle $\nu\left(M_{\psi}^{g}, M\right)$ vanishes, so does $\operatorname{Sign}(g, M)$.

This follows immediately from Theorem 4.21.

Corollary 4.24. In the setup of Theorem $4.22(\mathrm{i})$

$$
\sum_{\psi} \sum_{\left\{\omega \in \Omega\left(\psi^{\sigma}\right):\|\omega\|=d_{\psi}\right\}}(-1)^{\varepsilon\left(d, \mathbf{n}\left(\psi^{\sigma}\right), \omega\right)} \mathscr{S}_{\omega}\left(\nu_{\psi^{\sigma}}\right) \equiv 0 \quad(\bmod 3) .
$$

Denote by $\nu_{\psi}^{(2)}$ the $g$-invariant subbundle of $\nu_{\psi}$ corresponding to the eigenvalues $\left\{\lambda_{1 / m}^{j}\right\}$ with $j \equiv 2(\bmod 4)$. 
Corollary 4.25. Under the assumptions of Theorem 4.22(ii)

$$
\operatorname{Sign}(g, M) \equiv \sum_{\psi} \operatorname{Sign}\left(M_{\psi^{\sigma}}^{g} \pitchfork_{\nu_{\psi^{\sigma}}^{(2)}} M_{\psi^{\sigma}}^{g}\right) \quad(\bmod 2) .
$$

In particular, if for each $\psi$ the Euler class of $\nu_{\psi}^{(2)}$ vanishes, then $\operatorname{Sign}(g, M)$ is even.

In fact, all the terms on the right-hand side of the formula in Theorem 4.22(ii) are even, with the possible exception of those with $|\omega|=\left|\mathbf{n}^{(2)}\left(\psi^{\sigma}\right)\right|$, i.e., those $\omega \in \Omega^{(2)}\left(\psi^{\sigma}\right)$ satisfying $\omega_{j, k}=0, j \not \equiv 2(\bmod 4)$. For these exceptional $\omega$ 's we have $\mathscr{S}_{\omega}\left(\nu_{\psi^{\sigma}}\right)=\operatorname{Sign}\left(M_{\psi^{\sigma}}^{g} \pitchfork_{\nu_{\psi^{\sigma}}^{(2)}} M_{\psi^{\sigma}}^{g}\right)(\bmod 2)$. This proves the corollary.

Let us now apply Lemma 2.10(i) to the $\sigma J$-diagonal polynomial $P=\mathscr{N}_{\left(M, g^{\sigma}\right)}$ - $\operatorname{Sign}\left(g^{\sigma}, M\right)$ produced by a $\sigma$-concentrated action with $\operatorname{Sign}\left(g^{\sigma}, M\right) \in \mathbf{Z}$. It is readily verified that the leading homogeneous part of $P$ is

$$
{ }_{\mathbf{t}_{1}} P=\sum_{\psi} \sum_{\left\{\omega \in \Omega\left(\psi^{\sigma}\right):\|\omega\|=d_{\psi}\right\}}(-1)^{d_{\psi}}\left[\prod_{j \in\left[\psi^{\sigma}\right]} X_{j}^{n_{j}\left(\psi^{\sigma}\right)+\left\|_{j} \omega\right\|}\right] \mathscr{S}_{\omega}\left(\nu_{\psi^{\sigma}}\right) .
$$

Substituting $\left\{X_{j}=1 / j\right\}_{j \in \sigma J}$ we obtain

Proposition 4.26. In the setup of Theorem 4.20

$$
\sum_{\psi} \sum_{\left\{\omega \in \Omega\left(\psi^{\sigma}\right):\|\omega\|=d_{\psi}\right\}}(-1)^{d_{\psi}}\left[\prod_{j \in\left[\psi^{\sigma}\right]}(1 / j)^{n_{j}\left(\psi^{\sigma}\right)+\left\|_{j} \omega\right\|}\right] \mathscr{S}_{\omega}\left(\nu_{\psi^{\sigma}}\right)=0 .
$$

Remark 4.27. In [BK, Proposition 6.2] a modulo $p$ version of this result was obtained for general diffeomorphism of odd prime power order $p^{e}$.

Proposition 4.26 implies

Corollary 4.28. There exist no $\sigma$-concentrated diffeomorphism $g$ with a single fixed point on a closed manifold with $\operatorname{Sign}\left(g^{\sigma}, M\right) \in \mathbf{Z}$.

Corollary 4.29. Assume that $g: M \rightarrow M$ satisfies the hypotheses of Theorem 4.20 and that $\operatorname{codim}\left(M^{g}, M\right)=2$. Then

$$
\sum_{j \in \sigma J} j^{-d} \cdot \#\left(\pitchfork^{d} M_{j}^{g}\right)=0 .
$$

Again, this should be compared with its modulo $p$ analogue [BK, Proposition 8.1] (and with Corollary 4.13).

In the case where all normal bundles $\left\{\nu_{\psi}\right\}$ are equivariantly trivial, $\mathscr{S}_{\omega}\left(\nu_{\psi}\right)$ $=0$ for each $\omega \neq 0$. Combined with the fact that the summation in Proposition 4.26 extends only over $\omega$ 's with $\|\omega\|=d_{\psi}$, this shows that only the isolated fixed points have a nontrivial contribution to that sum. Thus, we have proved

Corollary 4.30. Let $g: M \rightarrow M$ be as in Theorem 4.20. If all normal bundles $\left\{\nu_{\psi}\right\}$ are equivariantly trivial, then

$$
\sum_{\left\{\psi: d_{\psi}=0\right\}} \prod_{j \in\left[\psi^{\sigma}\right]}(1 / j)^{n_{j}\left(\psi^{\sigma}\right)} \cdot \#\left(M_{\psi^{\sigma}}^{g}\right)=0
$$


Our last result will demonstrate that the techniques developed in this section are applicable to quite general infinite group actions. Let $G$ be a subgroup of the circle group $S^{1}$, endowed with the induced topology. By a $G$-action on a compact manifold $M$ we mean a continuous homomorphism $\Theta: G \rightarrow$ $\operatorname{Diff}(M)$, where $\operatorname{Diff}(M)$ is viewed as a topological group endowed with the uniform topology.

Theorem 4.31. All the results of this section (appropriately reformulated) are valid for any G-action on a closed even-dimensional manifold $M, G$ being an unbounded torsion subgroup of $S^{1}$ satisfying the following conditions:

(i) The G-action has only finitely many orbit types.

(ii) The G-action is linearizable near $M^{G}$, i.e., $M^{G}$ is a smooth submanifold of $M$ and the G-action on the normal bundle $\nu\left(M^{G}, M\right)$ is fiberpreserving and linear on each fiber.

In particular, this is the situation for any circle action $S^{1} \times M \rightarrow M, C^{2}$ smooth in both variables.

Proof. By continuity, each isotropy group $G_{x}, x \in M$, is either finite or $G$ itself. Using (i) we can pick a finite subgroup of $G$, say $C_{m}$, properly containing each isotropy group $G_{x}, x \in M \backslash M^{G}$. For such a choice of $m$, $M^{G}=M^{C_{m}}$. Now (ii) means that $G$ acts fiberwise linearly on $\nu\left(M^{G}, M\right)$. Again by continuity, which in this setup implies uniform continuity, this $G$ action can be extended to a continuous $S^{1}$-action, also acting linearly on the fibers of $\nu\left(M^{G}, M\right)=\nu\left(M^{S^{1}}, M\right)$ (it is not at all evident that the $G$-action on $M$ necessarily extends to an $S^{1}$-action). On every fiber the $S^{1}$-action is given by a block matrix, each block being of the form $\left(\begin{array}{c}\cos r \theta \sin r \theta \\ -\sin r \theta \cos r \theta\end{array}\right), r$ a nonzero integer, $e^{i \theta}$ a typical element of $S^{1}$. Obviously, $\nu\left(M^{S^{1}}, M\right)$ splits canonically into a direct sum $\bigoplus_{r>0}\left(\nu_{r} \oplus \nu_{-r}\right)$ of $S^{1}$-bundles, the $\nu_{r}$ 's corresponding to distinct positive $r$ 's. Writing $r=2^{h(r)} \cdot \tilde{r}$ with $(\tilde{r}, 2)=1$, one can introduce an equivariant complex structure in each $\nu_{r}$ given by the restriction at $\nu_{r}$ of the action of $\exp \left(i \pi / 2^{h(r)+1}\right)$. With respect to this structure, a typical normal $S^{1}$-representation $\Psi$ splits into a direct sum of complex 1-dimensional representations $\rho_{r}$ of the form $\rho_{r}\left(e^{i \theta}\right)=e^{i r \theta}$. Thus, we have finitely many $r$ 's produced from the various $\Psi$ 's. Replacing $m$ by a suitable multiple thereof, we may assume that distinct $r$ 's remain distinct modulo $m$ and that

$$
\operatorname{dim} M \cdot \sum_{r}|r|<2 \varphi(m) .
$$

Restricting the $G$-action to $C_{m}$ we get a smooth $C_{m}$-action at the bundle $\nu\left(M^{C_{m}}, M\right)$, possessing a continuous $C_{m}$-equivariant complex structure. By a standard approximation technique, it is possible to approximate (and therefore 
replace) this complex structure in $\nu\left(M^{C_{m}}, M\right)$ be a smooth $C_{m}$-equivariant one. Now Lemma 4.6 evidently implies that the restriction of the $G$-action to $C_{m}$ is 1-concentrated.

It is clear that both the $G$-action and the $C_{m}$-action determine the same canonical (smooth) splittings of $\nu\left(M^{G}, M\right)$ in accordance with the distinct "eigenvalues" of the restrictions of the $\Psi$ 's to $G$ and to $C_{m}$, respectively. Since normal quasi-signatures are defined only in terms of this splitting, and since distinct $r$ 's lie in distinct residue classes modulo $m$, the polynomial $\mathscr{N}_{(M, g)}$, $\left(g \in C_{m}\right)$, is in fact independent of the choice of such an $m$. Consequently we can define $\mathscr{N}_{(M, g)}$ by means of the $G$-action.

Since $M$ is a compact manifold, there exists an $\varepsilon>0$ such that any two $\varepsilon$-close maps from $M$ to $M$ are homotopic. It follows from the definition of a $G$-action that there exists a $\delta>0$ such that if $g \in G$ is $\delta$-close to $1 \in G$ in the metric induced on $G$ from $S^{1}$, then $\Theta(g)$ is $\varepsilon$-close to $\Theta(1)$ and thus homotopic to it. Given any $\delta$-close $g^{\prime}, g^{\prime \prime} \in G$, write $g^{\prime}=g_{0}$ and $g^{\prime \prime}=g_{0} g$. Since $\Theta\left(g_{0}\right)=\Theta\left(g_{0}\right) \cdot \Theta(1)$ and $\Theta\left(g_{0} g\right)=\Theta\left(g_{0}\right) \cdot \Theta(g)$, the homotopy between $\Theta(g)$ and $\Theta(1)$ produces a homotopy between $\Theta\left(g^{\prime \prime}\right)$ and $\Theta\left(g^{\prime}\right)$. $G$ being infinite, for any $h \in G$ there exists a finite sequence in $G$, "connecting" 1 with $h$, in which any two consecutive terms are $\delta$-close. Therefore, for any $h \in G$, $\Theta(h)$ is homotopically trivial, which implies that $\operatorname{Sign}(h, M)=\operatorname{Sign}(M) \in \mathbf{Z}$. This completes the proof.

Corollary 4.32. Let $G$ be an unbounded torsion subgroup of $S^{1}$ and $M$ a closed oriented smooth manifold. Then any smooth $G$-action on $M$ with finitely many orbit types has a fixed point, provided $\operatorname{Sign}(M) \neq 0$. If in addition, the action is linearizable at $M^{G}$, then it has at least $|\operatorname{Sign}(M)|$ fixed points. If $M^{G}$ is finite, then its cardinality has the same parity as $\operatorname{Sign}(M)$ (here $\operatorname{Sign}(M)$ may be 0$)$. When $\operatorname{Sign}(M)= \pm 1$, the action has at least three fixed points.

Proof. We may obviously assume that $M^{G}$ is finite. If $\operatorname{Sign}(M) \neq 0$, then Theorem 4.31, combined with Theorem 4.20 , implies that $M^{G}$ is nonempty. Since the signature of a singleton is \pm 1 , the aforementioned two theorems yield $\operatorname{card}\left(M^{G}\right) \geq|\operatorname{Sign}(M)|$ and $\operatorname{card}\left(M^{G}\right) \equiv \operatorname{Sign}(M)(\bmod 2)$. By Corollary 4.28, $G$ cannot have a single fixed point. Therefore, $\operatorname{Sign}(M)= \pm 1$ implies $\operatorname{card}\left(M^{G}\right) \geq 3$.

\section{REFERENCES}

[AH] J. P. Alexander and G. C. Hamrick, Periodic maps on Poincaré duality spaces, Comment. Math Helv. 53 (1978), 149-159.

[AS] M. F. Atiyah and I. M. Singer, The index of elliptic operators. III, Ann. of Math. 87 (1968), 546-604.

[BK] D. Berend and G. Katz, Separating topology and number theory in the Ativah-Singer $g$ signature formula, Duke Math. J. 61 (1990). 
[H] F. Hirzebruch, Topological methods in algebraic geometry, Springer-Verlag, New York, 1966.

[KR] K. Kawakubo and F. Raymond, The index of manifolds with toral actions and geometric interpretations of the $\sigma\left(\infty,\left(S^{1}, M^{n}\right)\right)$-invariant of Atiyah and Singer, Lecture Notes in Math., vol. 298, Springer-Verlag, pp. 228-233.

Department of Mathematics and Computer Science, Ben-Gurion University of the Negev, Beer-Sheva 84105, Israel (Current address of Daniel Berend)

Current address (Gabriel Katz): Department of Mathematics, Florida Atlantic University, Boca Raton, Florida 33431 